\title{
Robust Position Control of a Two-Sided 1-DoF Impacting Mechanical Oscillator Subject to an External Persistent Disturbance by Means of a State-Feedback Controller
}

\author{
Firas Turki, ${ }^{1}$ Hassène Gritli $\mathbb{D}^{1,2}$ and Safya Belghith ${ }^{1}$ \\ ${ }^{1}$ Laboratory of Robotics, Informatics and Complex Systems (RISC-Lab, LR16ES07), National Engineering School of Tunis, \\ Université de Tunis El Manar, BP. 37, Le Belvédère, 1002 Tunis, Tunisia \\ ${ }^{2}$ Higher Institute of Information and Communication Technologies, Université de Carthage, 1164 Borj-Cedria, Tunis, Tunisia
}

Correspondence should be addressed to Hassène Gritli; grhass@yahoo.fr

Received 6 September 2019; Accepted 1 November 2019; Published 7 December 2019

Guest Editor: Alberto Luviano

Copyright (c) 2019 Firas Turki et al. This is an open access article distributed under the Creative Commons Attribution License, which permits unrestricted use, distribution, and reproduction in any medium, provided the original work is properly cited.

This paper proposes a state-feedback controller using the linear matrix inequality (LMI) approach for the robust position control of a 1-DoF, periodically forced, impact mechanical oscillator subject to asymmetric two-sided rigid end-stops. The periodic forcing input is considered as a persistent external disturbance. The motion of the impacting oscillator is modeled by an impulsive hybrid dynamics. Thus, the control problem of the impact oscillator is recast as a problem of the robust control of such disturbed impulsive hybrid system. To synthesize stability conditions, we introduce the S-procedure and the Finsler lemmas by only considering the region within which the state evolves. We show that the stability conditions are first expressed in terms of bilinear matrix inequalities (BMIs). Using some technical lemmas, we convert these BMIs into LMIs. Finally, some numerical results and simulations are given. We show the effectiveness of the designed state-feedback controller in the robust stabilization of the position of the impact mechanical oscillator under the disturbance.

\section{Introduction}

In diverse mechanical systems, there are some working conditions and parameters leading to the exhibition of impacts between the oscillatory elements of certain mechanical systems due to the presence of gaps. Some impacting mechanical systems are the impact dampers, the inertial shakers, the impact print hammers, the pile drivers, the shock absorbers, the forming machines, and much more [1-3]. In one-degree-of-freedom (1-DoF) mechanical oscillators, impacts can occur with rigid/soft end-stops, whereas in multi-DoF oscillators, collisions can also occur amongst moving elements. The wide interest in these impacting systems has spurred researchers and engineers to analyze their motions and hence the implications of impacts, as, for example, in [3-17].

In some working cases, impacts between interacting bodies are considered intrinsic for the operations of many engineering devices. Nevertheless, impacts may provoke some dangerous effects such as impulsive severe forces, rapid transfer of energy, and stresses and provokes a degradation of the performance of the mechanical systems, where we expect to observe a smooth behavior $[2,3,7,8,12,14]$. These effects can lead then to the exhibition of chaotic responses. Thus, the objective is to improve the response and the performances of these impacting mechanical systems through control. The motion of these impacting systems is governed by an impulsive hybrid dynamics, which is composed by a differential equation and an algebraic one associated with its impact conditions. Thus, due to this impulsive hybrid dynamics, which is considered complex enough for analysis and control, the synthesis of controllers for these impacting systems is difficult to realize.

Some control techniques can be found in the literature. The OGY method has been widely adopted and applied to control chaos in vibroimpact systems [13, 18-22]. Some 
other authors, e.g., [23-27], used other control techniques such as an external driving force, a delay feedback, a displacement feedback, a discrete-in-time feedback control, and a damping control law, among others. Authors in [28] used a feedback control input to control the position of a 1DoF impact mechanical oscillator with two asymmetric endstops. Authors in [29] controlled chaos in a 1-DoF impact oscillator with only one rigid constraint.

In this paper, we consider the control problem of the position of a 1-DoF impact mechanical oscillator with twosided rigid end-stops [28, 30-32]. Such an impacting oscillator is periodically forced via an external sinusoidal input, which will be considered as a persistent disturbance to compensate its effect via a robust control law. Our main idea lies first in adding a control input to the impact oscillator. The synthesis of the control law will be realized using the LMI approach. In this paper, we will consider the whole impulsive hybrid dynamics of the two-sided 1-DoF impacting oscillator. Both the differential equation and the algebraic equations are considered in the design of the stability condition. In our previous works [30-32], the impact dynamics was not taken into account in the design. Only the differential equation was considered. Moreover, we adopted a state-feedback control law to stabilize the impacting motion around the zero-equilibrium point. As in [28], the forcing input, as a known signal, was canceled via the controller [30]. However, in [31, 32], the forcing input was considered as a disturbance. In this work, we will adopt a state-feedback controller to robustly stabilize the impulsive hybrid dynamics of the two-sided 1-DoF impact oscillator.

In order to synthesize the stability conditions, we use first the S-procedure lemma in order to only take into consideration the working region of the two-sided impacting oscillator, that is, the region between the two end-stops. In addition, we use the Finsler lemma in order to develop stability conditions for the algebraic equation and using the impact conditions. We show then that these stability conditions are expressed in terms of bilinear matrix inequalities (BMIs). In order to obtain numerically traceable LMI conditions, we use mainly the Schur complement and the matrix inversion lemma. Authors in $[33,34]$ used the LMI approach and these previous lemmas, except the Finsler Lemma, in order to design stabilizing controllers for an underactuated mechanical system, namely, the inertia wheel inverted pendulum, for which its motion is constrained by two symmetric end-stops. Its dynamics is nonlinear and does not present impacts. Our objective in this paper is to extend the design methodology adopted in $[33,34]$ for the two-sided 1-DoF impact mechanical oscillator, which is characterized by an impulsive hybrid dynamics.

The main contributions of this paper are summarized as follows:

(i) A robust state-feedback control law is designed to stabilize the 1-DoF double-sided mechanical oscillator subject to two asymmetric rigid constraints and an external persistent disturbance.

(ii) Stabilization of the impulsive hybrid dynamics of the impacting oscillator, where the continuous dynamics and the impact dynamics are both considered in the synthesis of the stability conditions.

(iii) Via the S-procedure and Finsler lemmas, the stabilization conditions of the closed-loop impulsive hybrid system are first obtained and expressed in terms of BMIs. The stability conditions are considered to be only satisfied inside the working region.

(iv) By means of the Schur complement lemma and the matrix inversion lemma, the previous BMIs are converted into LMIs.

(v) Several simulations are presented to show that the designed robust control law is able to compensate the effect of the external persistent disturbance and hence stabilize the impact mechanical oscillator at the zero-equilibrium point.

The structure of this paper is as follow. In Section 2, the two-sided 1-DoF impact mechanical oscillator is presented. Its impulsive hybrid dynamics is also presented in this section. The problem formulation and the technical lemmas used through this paper are given in Section 3. Section 4 and Section 5 are devoted to the synthesis of the BMI and the LMI stability conditions, respectively. In Section 6, the numerical results and simulations are demonstrated. Finally, the conclusion is addressed in Section 7.

\section{The Double-Sided 1-DoF Impact Mechanical Oscillator}

2.1. System Description. Figure 1 shows a $1-\mathrm{DoF}$ impact mechanical oscillator with asymmetric double-sided rigid end-stops. The impacting system is composed of a mass $m$ interconnected to the right end-stop by both a spring of stiffness $k$ and a dashpot of damping coefficient $\zeta$. The gap between the mass $m$ and the rigid stop is $d$. A second rigid end-step is localized at the left away from the mass of a distance $l$. The motion impact oscillator is excited via a sinusoidal force input $v=p \cos (w t)$, where the parameters $w$ and $p$ are the excitation frequency and the excitation amplitude, respectively. In Figure 1, $u$ is an additional input, the controller, which will be designed next to control the position of the impact oscillator to the zero-equilibrium point.

From an initial condition, the mechanical oscillator will oscillate horizontally along the axis $x$ and will produce impacts with the two asymmetric rigid constraints with the same coefficient $r$ of restitution inducing hence an instantaneous transition of the velocity.

2.2. Impulsive Hybrid Dynamics of the Impact Oscillator. The dynamics of the two-sided 1-DoF impact mechanical oscillator is governed by the following system:

$$
\begin{array}{ll}
m \ddot{\mathbf{x}}+2 \zeta \dot{\mathbf{x}}+k \mathbf{x}=p \cos (w t), & \text { for } l<\mathbf{x}<d, \\
\mathbf{x}^{+}=\mathbf{x}^{-} \text {and } \dot{\mathbf{x}}^{+}=-r \dot{\mathbf{x}}^{-}, & \text {for } \mathbf{x}=d \text { or } \mathbf{x}=l,
\end{array}
$$




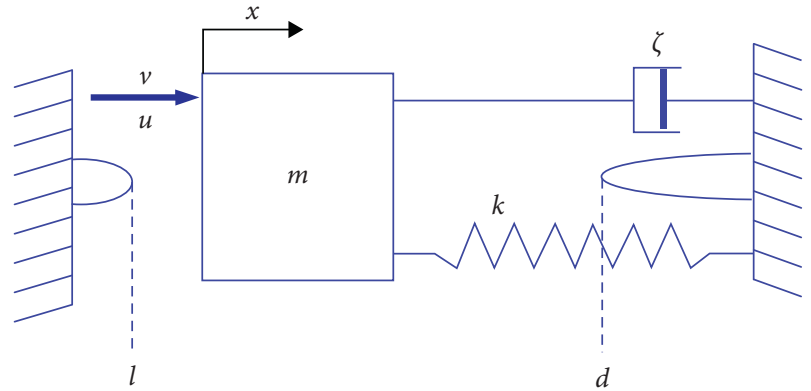

FIgURE 1: A 1-DoF impact mechanical oscillator under two asymmetric rigid end-stops.

where $\ddot{\mathbf{x}}, \dot{\mathbf{x}}$, and $\mathbf{x}$ are the acceleration, velocity, and displacement of the mass of the impacting oscillator, $d$ and $l$ are the right bound and the left bound, respectively, and subscribes $^{+}$and $^{-}$in (1b) denote, respectively, just after and just before the impact.

2.3. A Brief Look on the Impacting Behavior. It is well known that the double-sided 1-DoF impact mechanical oscillator generates periodic and chaotic behaviors with respect to the parameters $p, w, \zeta, d, l$, and $r$. We refer our reader to [28] for further details and some bifurcation diagrams. As in [28], the adopted values of different parameters in dynamics (1a) and (1b) are $m=1 \mathrm{~kg}, \quad k=1 \mathrm{~N} / \mathrm{m}, \quad \zeta=0.05 \mathrm{~N} /(\mathrm{m} / \mathrm{s})$, $d=0.3 \mathrm{~m}, l=-0.1 \mathrm{~m}, p=0.5 \mathrm{~N}$, and $r=0.8$. Figure 2 shows the behavior of the impact oscillator for $w=0.8$. In Figure 2, we plotted the temporal evolution of the position and the velocity of the impacting mechanical system. Figure 3 reveals the corresponding phase portrait simulated for such value of the parameter $w$. In Figure 4, we presented three Poincaré maps. Figure 4(a) shows the stroboscopic Poincare map, which reveals the state of the impact oscillator at the period T. Figures 4(b) and 4(c) reveal the impact Poincaré maps. The first one in Figure 4(b) shows the impact time of the oscillator with the right bound with respect to its impact velocity. However, the second impact Poincaré map in Figure 4(c) shows the impact time with the left rigid constraint with respect to the impact velocity. These previous portraits in Figures 2, 3, and 4 demonstrate that the 1-DoF impact mechanical oscillator displays a chaotic behavior.

It is worth to note that it is possible to characterize chaos exhibited in the motion of the 1-DoF impact mechanical oscillator presented in Figure 2 and then in Figures 3 and 4 by means of the spectrum of Lyapunov exponents. Authors in [35] computed numerically the Lyapunov exponents in mechanical systems with impacts using a transcendental map. They applied their computation procedure for the onesided 1-DoF impact oscillator and also for the impact-pair system. Moreover, authors in [36] computed the Lyapunov exponents of a cantilever beam impacting on a moving base using a discrete modeling. Some other works have also been developed for this subject, as, e.g., in [37, 38]. Author in [39] presented a general method for the calculation of Lyapunov exponents for dynamic systems with discontinuities and then with impacts. Thus, a forced impact oscillator with dry

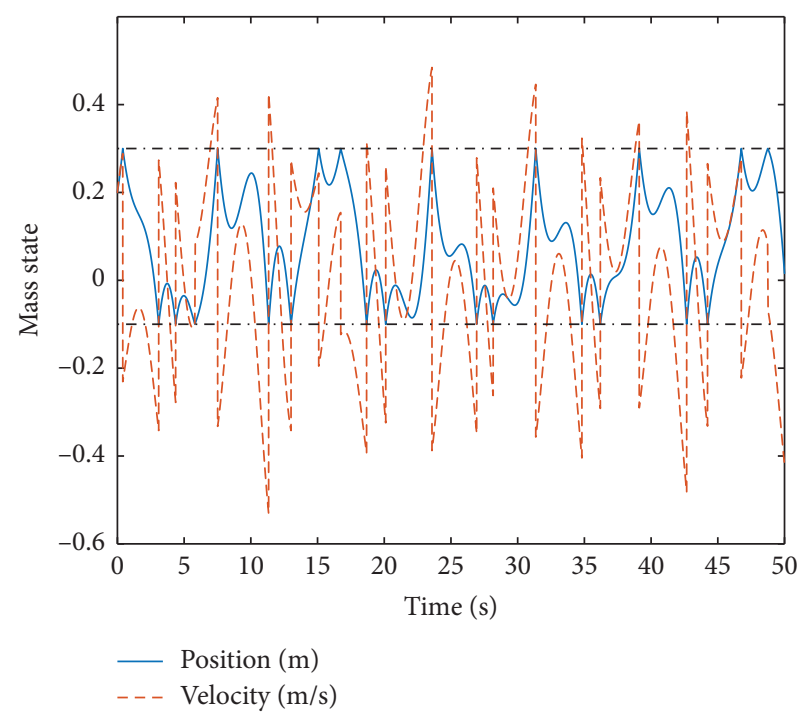

FIgURE 2: Temporal evolution of the mass position of the doublesided 1-DoF impacting mechanical oscillator for $w=0.8$.

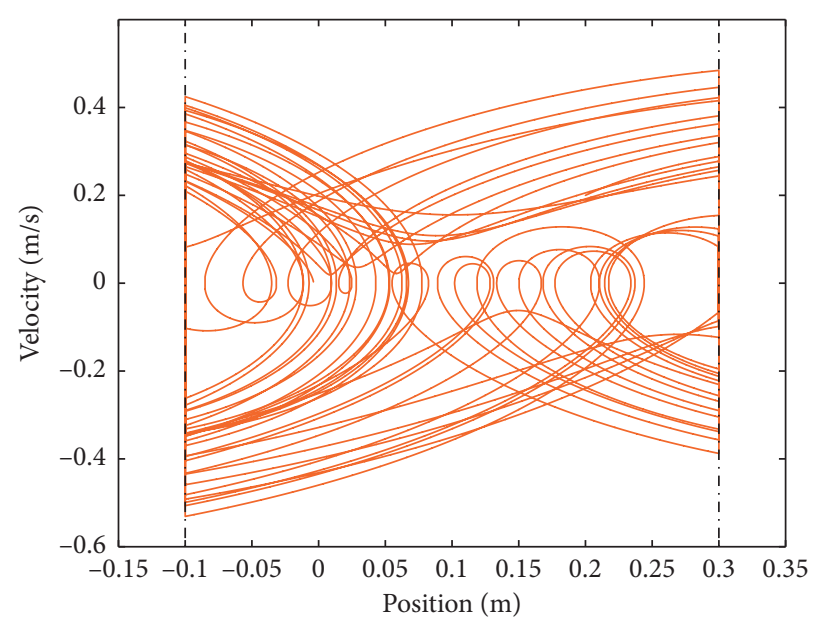

Figure 3: Phase portrait of the double-sided 1-DoF impacting mechanical oscillator for the parameter $w=0.8$ showing a chaotic attractor.

friction was presented as an illustrative example. In [40, 41], authors computed the spectrum of Lyapunov exponents for more complicated impulsive hybrid systems, namely, the biped robots, using an explicit analytical expression of the (hybrid) Poincaré map.

\section{Problem Formulation and Preliminaries}

3.1. Problem Statement. Let $\mathbf{z}=\left[\begin{array}{ll}x & \dot{x}\end{array}\right]^{T}$ be the state vector. Then, relying on the equations in (1a) and (1b), the impulsive hybrid dynamics of the 1-DoF impact mechanical oscillator under the double-sided rigid constraints is given as follows:

$$
\begin{aligned}
& \dot{\mathbf{z}}=\mathscr{A} \mathbf{z}+\mathscr{B} v \text {, as long as } l<\mathscr{C}^{T} \mathbf{z}<d, \\
& \mathbf{z}^{+}=\mathscr{R} \mathbf{z}^{-}, \text {whenever } \mathscr{C}^{T} \mathbf{z}=d \text { or } \mathscr{C}^{T} \mathbf{z}=l,
\end{aligned}
$$




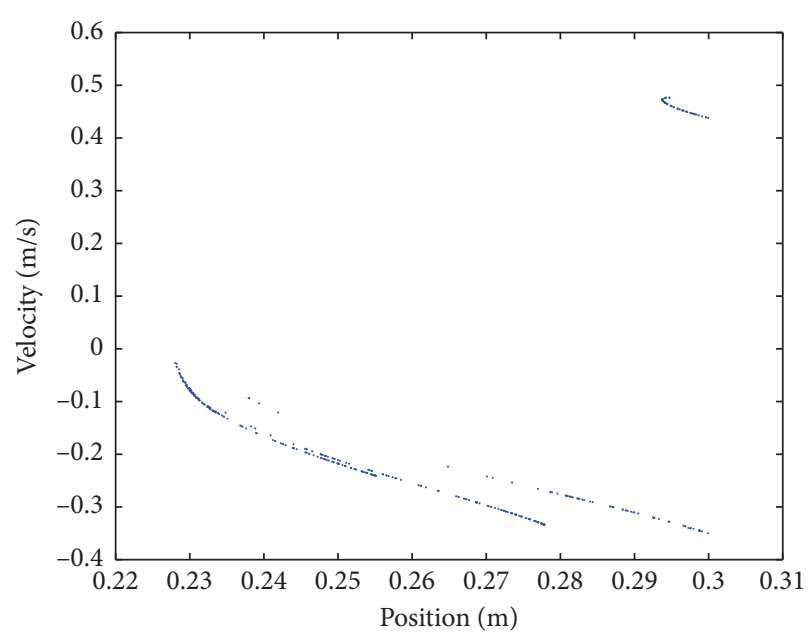

(a)

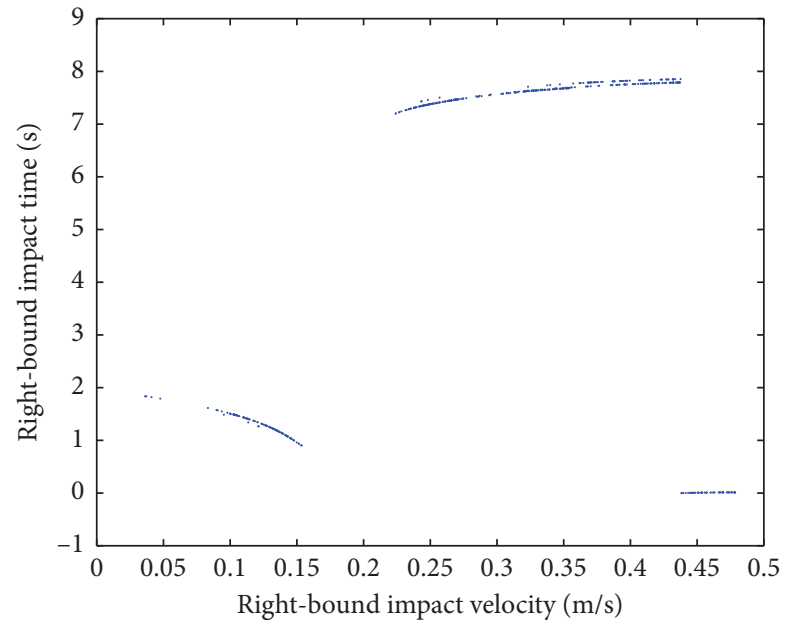

(b)

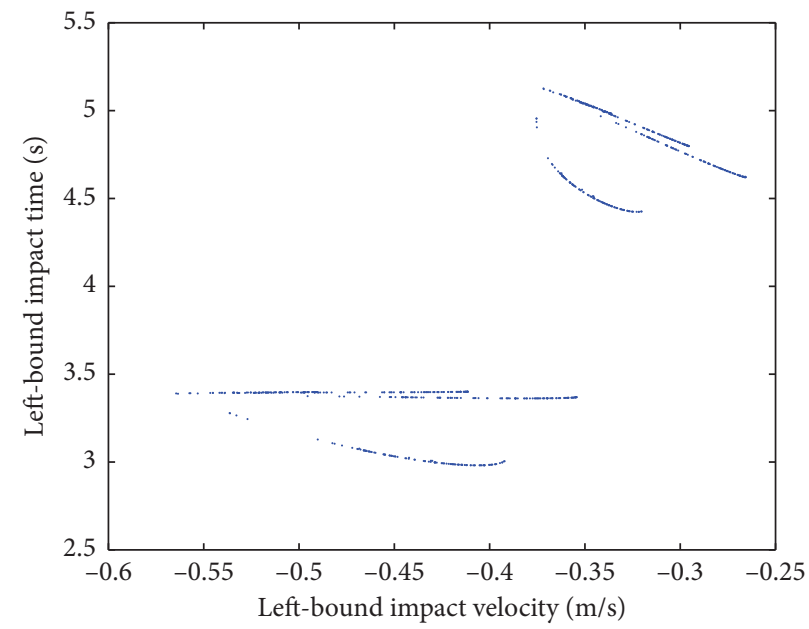

(c)

FIGURE 4: Phase portrait of the double-sided 1-DoF impacting mechanical oscillator for the parameter $w=0.8$ showing a chaotic attractor.

where $\quad \mathscr{A}=\left[\begin{array}{cc}0 & 1 \\ -(k / m) & -(2 \zeta / m)\end{array}\right], \quad \mathscr{B}=\left[\begin{array}{c}0 \\ 1 / m\end{array}\right]$, $\mathscr{R}=\left[\begin{array}{cc}1 & 0 \\ 0 & -r\end{array}\right], \mathscr{C}=\left[\begin{array}{l}1 \\ 0\end{array}\right]$, and $v=p \cos (w t)$. Moreover, we note that $d>0$ and $l<0$.

Our main objective in this work is to control the position of the impacting oscillator to the zero-equilibrium point. Then, we need an additional external input, saying $u$, as the controller, which will be designed next. Moreover, the signal $v$ will be considered next as an external disturbance input applied to the oscillator. Actually, we will consider a general form of the disturbance torque $v$ that can take any expression such that $|v| \leq p$. Accordingly, in (2a), we will have $(u+v)$ instead of $v$. Thus, the impulsive hybrid dynamics (1a) and (1b) becomes

$$
\begin{aligned}
\dot{\mathbf{z}} & =\mathscr{A} \mathbf{z}+\mathscr{B} u+\mathscr{B} v, \text { as long as } \mathbf{z} \in \Omega, \\
\mathbf{z}^{+} & =\mathscr{R} \mathbf{z}^{-}, \text {whenever } \mathbf{z} \in \Gamma
\end{aligned}
$$

In (3a) and (3b), $\Gamma$ represents the impact surface and $\Omega$ reveals the oscillation space. They are defined as follows:

$$
\begin{aligned}
\Omega & =\left\{\mathbf{z} \in \mathbb{R}^{2 \times 1}, l<\mathscr{C}^{T} \mathbf{z}<d\right\}, \\
\Gamma & =\left\{\mathbf{z} \in \mathbb{R}^{2 \times 1}, \mathscr{C}^{T} \mathbf{z}=d \text { or } \mathscr{C}^{T} \mathbf{z}=l\right\} .
\end{aligned}
$$

Our objective in this work is then to design the controller $u$ for the disturbed impulsive hybrid systems (3a) and (3b)-(4) of the 1-DoF impacting mechanical oscillator to robustly stabilize it at (around) the zero-equilibrium point even in the presence of the external disturbance input $v$. For this subject we assume that all the states, i.e., the position $x$ and the velocity $\dot{x}$ of the mechanical system are available for measurement. Then, under this assumption, we will adopt the following state-feedback controller:

$$
u= \begin{cases}\mathscr{K} \mathbf{z}, & \text { whenever } \mathbf{z} \in \Delta, \\ 0, & \text { elsewhere }\end{cases}
$$

with 


$$
\Delta=\left\{x \in \mathbb{R}, x^{2}>\varepsilon\right\},
$$

where $\mathscr{K} \in \mathbb{R}^{1 \times 2}$ is a constant gain matrix to design next using the LMI approach and $\varepsilon$ is a small enough positive constant. The set $\Delta$ in (6) defines the region within which the state-feedback control law is activated. This set $\Delta$ can be recast as follows:

$$
\Delta=\left\{\mathbf{z} \in \mathbb{R}^{2 \times 1}, \mathbf{z}^{T} \mathscr{C} \mathscr{C}^{T} \mathbf{z}>\varepsilon\right\} .
$$

Remark 1. It is worth to note that the condition $\mathbf{z}^{T} \mathscr{C} \mathscr{C}^{T} \mathbf{z}>\mathcal{E}$ defining the set $\Delta$ in expression of the state-feedback control law (5) was added in order to overcome a certain solving problem of the LMI-based stability conditions of the closedloop impulsive hybrid systems (3a) and (3b) under the state constraints in (4) using the Lyapunov approach [33]. Such a problem will be discussed later (see Remark 4). In [33], authors used the constraint $\|\mathbf{z}\|^{2}>\varepsilon$ on the state-feedback control law in (5). This last condition is identical to the constraint $\mathbf{z}^{T} \mathscr{C}^{T} \mathbf{z}>\varepsilon$ if $\mathscr{C}=\mathscr{I}_{2}$. We emphasize that the new condition $\mathbf{z}^{T} \mathscr{C} \mathscr{C}^{T} \mathbf{z}>\varepsilon$ in (7) will provide less restrictive LMI conditions compared with that obtained via the condition $\mathbf{z}^{T} \mathbf{z}>\boldsymbol{\varepsilon}$.

By substituting expression of control law (5) in dynamic model (3a), we obtain then the following closed-loop impulsive hybrid dynamics:

$$
\begin{aligned}
\dot{\mathbf{z}} & =(\mathscr{A}+\mathscr{B} \mathscr{K}) \mathbf{z}+\mathscr{B} v, \text { as long as } \mathbf{z} \in \Omega, \mathbf{z} \in \Delta, \\
\mathbf{z}^{+} & =\mathscr{R} \mathbf{z}^{-}, \text {whenever } \mathbf{z} \in \Gamma, \mathbf{z} \in \Delta .
\end{aligned}
$$

Remark 2. It is worth to note that in [28], the position control of the double-sided 1-DoF impacting mechanical oscillator was achieved using the following control law:

$$
u=2 \zeta \dot{x}+k x-v(t)-m(a x+b \dot{x}),
$$

where the two design parameters $a$ and $b$ are determined such that the eigenvalues of the matrix $\left[\begin{array}{cc}0 & 1 \\ -a & -b\end{array}\right]$ are with negative real parts.

In the next part of this work, we will focus on synthesizing stability conditions for the closed-loop impulsive hybrid systems (8a) and (8b).

3.2. Preliminary Lemmas. Before synthesizing the stability conditions for the impulsive hybrid system (8a) and (8b), we need the following technical lemmas. In the sequel, and in large matrix expressions, the symbol $(\star)$ replaces terms that are induced by symmetry. Moreover, $\boldsymbol{\Omega}+\boldsymbol{X}+(\star)=$ $\Omega+\mathscr{X}+\mathscr{X}^{\mathrm{T}}$. In addition, $\mathscr{I}$ and $\mathcal{O}$ stand, respectively, for the identity matrix and the null matrix with appropriate dimensions.

Lemma 1 (the Young inequality [42]). For given constant matrices $\mathbf{X}$ and $\mathbf{Y}$ with appropriate dimensions and any positive scalar $\lambda$, the following inequality holds:

$$
\mathbf{X}^{T} \mathbf{Y}+\mathbf{Y}^{T} \mathbf{X} \leq \lambda \mathbf{X}^{T} \mathbf{X}+\lambda^{-1} \mathbf{Y}^{T} \mathbf{Y}
$$

Lemma 2 (the matrix inversion lemma [33, 34]). For given invertible matrices $\mathbf{X}$ and $\mathbf{Y}$ such that $\mathbf{X} \in \mathbb{R}^{n \times n}$ and $\mathbf{Y} \in \mathbb{R}^{m \times m}$. Moreover, given matrices $\mathbf{U}$ and $\mathbf{V}$ are of appropriate dimensions $\mathbf{U} \in \mathbb{R}^{n \times m}$ and $\mathbf{V} \in \mathbb{R}^{m \times n}$. Then, the matrix inversion lemma is

$$
(\mathbf{X}+\mathbf{U Y V})^{-1}=\mathbf{X}^{-1}-\mathbf{X}^{-1} \mathbf{U}\left(\mathbf{Y}^{-1}+\mathbf{U X}^{-1} \mathbf{U}\right)^{-1} \mathbf{V} \mathbf{X}^{-1} .
$$

Lemma 3 (the Schur complement lemma [42]). For given matrices $\mathbb{Q}=\mathfrak{Q}^{T}, \mathscr{R}=\mathscr{R}^{T}$, and $\mathcal{S}$ with appropriate dimensions, the following propositions are equivalent:

$$
\begin{gathered}
{\left[\begin{array}{cc}
\mathscr{Q} & \mathcal{S} \\
\mathcal{S}^{T} & \mathscr{R}
\end{array}\right]>0,} \\
\left\{\begin{array}{l}
\mathscr{R}>0, \\
\mathbb{Q}-\mathcal{S}^{-1} \mathcal{S}^{T}>0 .
\end{array}\right.
\end{gathered}
$$

Lemma 4 (the S-procedure lemma $[33,34,42]$ ). Let $\mathscr{F}_{0}, \ldots, \mathscr{F}_{p} \in \mathbb{R}^{n \times n}$ be symmetric matrices. We consider the following condition on $\mathscr{F}_{0}, \ldots, \mathscr{F}_{p}$ :

$$
\zeta^{T} \mathscr{F}_{0} \zeta>0 \text { for all } \zeta \neq 0 \text {, such that } \zeta^{T} \mathscr{F}_{i} \zeta \geq 0, \quad i=1, \ldots, p \text {. }
$$

If there exist scalar variables $\tau_{1} \geq 0, \ldots, \tau_{p} \geq 0$, such that

$$
\mathscr{F}_{0}-\sum_{i=1}^{p} \tau_{i} \mathscr{F}_{i}>0
$$

then (13) holds.

Lemma 5 (Finsler's lemma [43]). Let, $x \in \mathbb{R}^{n}, \mathbb{Q}=\mathbb{Q}^{T} \in \mathbb{R}^{n \times n}$, and $\mathscr{B} \in \mathbb{R}^{n \times n}$ such that $\operatorname{rank}(B)<n$. The following statements are equivalent:

(i) $x^{T} \mathscr{Q} x<0$, for all $\mathscr{B} x=0, x \neq 0$

(ii) $\exists \mu \in \mathbb{R}: \mathbb{Q}-\mu \mathscr{B}^{T} \mathscr{B}<0$

Remark 3. In this paper, we will use the LMI approach to cope with the control problem of the 1-DoF impact mechanical oscillator, which is described by a second-order dynamics. We will design a robust control law in order to stabilize the position of such mechanical systems with impulse effects. Actually, the presence of an impulsive effect can influence the behavior of the solutions and cause dangerous effects in some applications. More than that, the presence of impulsive effects can cause instability of the whole behavior of the impacting mechanical system. These systems are defined by an impulsive hybrid dynamics for which the system undergoes a very instantaneous transition when reaching some state-dependent conditions, as in our case for the two-sided impact mechanical oscillator. Then, in this 
paper, we deal with an impulsive hybrid system, which is more complicated than a simple nonlinear system.

In addition, we stress that we deal with homogeneous models (3a) and (3b) allowing the interaction between discrete and continuous parts and the stabilization process was realized for whole models (8a) and (8b). Only the continuous part/model (3a) was found to be under control, whereas the discrete model (3b) or ( $8 b)$ is without control and also is unstable (the two eigenvalues of the matrix $\mathscr{R}$ are 1 and $-r$ ). Hence, the main challenge of the present work is to stabilize robustly the whole impulsive hybrid dynamics using a single controller for the continuous part.

Furthermore, to design the stability conditions of the closed-loop systems (8a) and (8b), we will only consider the working region, inside which the state vector is defined and then the impact mechanical oscillator evolved. Actually, we have two working regions: one for the continuous part and another for the discrete part. For the continuous model, the working region is that between the two asymmetric rigid end-stops, that is, the set $\Omega$ defined by (4a). However, for the discrete model ( $8 b)$, the working region is the two end-stops, which are defined by the impact condition and then set $\Gamma$ defined by (4b). We will use the S-procedure lemma to deal with the working region $\Omega$ and the Finsler Lemma to consider the working region $\Gamma$ for the design of the stability conditions.

\section{Synthesis of the Robust State-Feedback Control Law: BMI Conditions}

In order to synthesize stability conditions of the disturbed impulsive hybrid systems (8a) and (8b), we adopt the following Lyapunov function:

$$
\mathscr{V}(\mathbf{z})=\mathbf{z}^{T} \mathscr{P} \mathbf{z}
$$

where $\mathscr{P}=\mathscr{P}^{T}$.

Thus, the asymptotic stability conditions of systems (8a) and $(8 b)$ are given by

$$
\begin{array}{rr}
\mathscr{V}(\mathbf{z})>0 & \text { s.t. } \mathbf{z} \in \Omega, \mathbf{z} \in \Delta, \\
\dot{\mathscr{V}}(\mathbf{z})<0, & \text { s.t. } \mathbf{z} \in \Omega, \mathbf{z} \in \Delta, \\
\mathscr{V}\left(\mathbf{z}^{+}\right)-\mathscr{V}\left(\mathbf{z}^{-}\right)<0, & \text { s.t. } \mathbf{z} \in \Gamma, \mathbf{z} \in \Delta .
\end{array}
$$

Theorem 1. The closed-loop impulsive hybrid systems (8a) and (8b) subject to the external disturbance $v$, with $\|v\| \leq p$, is asymptotically and robustly stable if for some fixed parameter $0<\varepsilon \ll 1$, there exist a symmetric matrix $\mathscr{P}$, a matrix $\mathscr{K}$, positive scalars $\alpha_{1}, \alpha_{2}, \alpha_{3}, \alpha_{4}, \beta_{1}, \beta_{2}, \beta_{3}, \beta_{4}$, and $\lambda$, and free two scalars $\xi_{1}$ and $\xi_{2}$ such that the following matrix inequalities are feasible:

$$
\begin{aligned}
& {\left[\begin{array}{cc}
\mathscr{P}-\beta_{3} \mathscr{C}_{\mathscr{C}^{T}} & \left(\beta_{1}-\beta_{2}\right) \mathscr{C} \\
(\star) & \epsilon \beta_{3}-2 \beta_{1} d+2 \beta_{2} l
\end{array}\right]>0} \\
& {\left[\begin{array}{cc}
\mathscr{P}(\mathscr{A}+\mathscr{B} \mathscr{K})+(\star)+\lambda \mathscr{P} \mathscr{B} \mathscr{B}^{T} \mathscr{P}+\alpha_{3} \mathscr{C}^{T} & -\left(\alpha_{1}-\alpha_{2}\right) \mathscr{C} \\
(\star) & \frac{p^{2}}{\lambda}-\varepsilon \alpha_{3}+2 \alpha_{1} d-2 \alpha_{2} l
\end{array}\right]<0,} \\
& {\left[\begin{array}{cc}
\mathscr{R} \mathscr{P} \mathscr{R}-\mathscr{P}-\xi_{1} \mathscr{C}_{\mathscr{C}^{T}+\alpha_{4} \mathscr{C}^{T}} & \xi_{1} d \mathscr{C} \\
(\star) & -\alpha_{4} \varepsilon-\xi_{1} d^{2}
\end{array}\right]<0,} \\
& {\left[\begin{array}{cc}
\mathscr{R} \mathscr{P} \mathscr{R}-\mathscr{P}-\xi_{2} \mathscr{C} \mathscr{C}^{T}+\beta_{4} \mathscr{C} \mathscr{C}^{T} & \xi_{2} l \mathscr{C} \\
(\star) & -\beta_{4} \varepsilon-\xi_{2} l^{2}
\end{array}\right]<0 .}
\end{aligned}
$$

$$
\begin{aligned}
& \mathscr{W}_{4}(\mathbf{z})=\left[\begin{array}{ll}
\mathscr{C}^{T} & -d
\end{array}\right]\left[\begin{array}{l}
\mathbf{z} \\
1
\end{array}\right]=0, \\
& \mathscr{W}_{5}(\mathbf{z})=\left[\begin{array}{ll}
\mathscr{C}^{T} & -l
\end{array}\right]\left[\begin{array}{l}
\mathbf{z} \\
1
\end{array}\right]=0 .
\end{aligned}
$$

The derivative of the Lyapunov function (15) is given as follows:

$$
\dot{\mathscr{V}}(\mathbf{z})=2 \mathbf{z}^{T} \mathscr{P}(\mathscr{A}+\mathscr{B} \mathscr{K}) \mathbf{z}+2 \mathbf{z}^{T} \mathscr{P} \mathscr{B} v .
$$

Relying on the Young relation (Lemma 1) and as $|v| \leq p$, we obtain the following condition: 


$$
2 \mathbf{z}^{T} \mathscr{P} \mathscr{B} v \leq \lambda \mathbf{z}^{T} \mathscr{P} \mathscr{B} \mathscr{B}^{T} \mathscr{P} \mathbf{z}+\frac{p^{2}}{\lambda},
$$

with $\lambda>0$.

By substituting expression (20) in relation (19), then the stability condition becomes

$$
\mathcal{U}(\mathbf{z})=\left[\begin{array}{c}
\mathbf{z} \\
1
\end{array}\right]^{T}\left[\begin{array}{cc}
\mathscr{P}(\mathscr{A}+\mathscr{B} \mathscr{K})+(\star)+\lambda \mathscr{P} \mathscr{B} \mathscr{B}^{T} \mathscr{P} & \mathscr{O} \\
(\star) & \frac{p^{2}}{\lambda}
\end{array}\right]\left[\begin{array}{c}
\mathbf{z} \\
1
\end{array}\right]<0 .
$$

In addition, using the algebraic equation in (8b), then the stability condition in (16c) becomes

$$
\mathscr{W}(\mathbf{z})=\mathbf{z}^{T}(\mathscr{R} \mathscr{P} \mathscr{R}-\mathscr{P}) \mathbf{z}<0 .
$$

Accordingly, based on the previous expressions, the stability conditions in (16a)-(16c) are reformulated as follows:

$$
\begin{gathered}
\mathscr{V}(\mathbf{z})>0, \quad \text { s.t. } \mathscr{W}_{1}(\mathbf{z})<0, \mathscr{W}_{2}(\mathbf{z})<0, \mathscr{W}_{3}(\mathbf{z})<0, \\
\mathcal{U}(\mathbf{z})<0, \quad \text { s.t. } \mathscr{W}_{1}(\mathbf{z})<0, \mathscr{W}_{2}(\mathbf{z})<0, \mathscr{W}_{3}(\mathbf{z})<0, \\
\mathscr{W}(\mathbf{z})<0, \quad \text { s.t. } \mathscr{W}_{4}(\mathbf{z})=0 \text { or } \mathscr{W}_{5}(\mathbf{z})=0, \mathscr{W}_{3}(\mathbf{z})<0 .
\end{gathered}
$$

Relying on the S-procedure lemma (see Lemma 4) and based on the Finsler lemma (see Lemma 5), the conditions in (23a)(23c) are equivalent, respectively, to the matrix inequalities in (17a)-(17d), for some positive scalars $\alpha_{i}$ and $\beta_{i}, i=1,2,3,4$, and $\xi_{1}, \xi_{2} \in \mathbb{R}$. This completes the proof of Theorem 1 .

Remark 4. It is worth to note that if we choose $\varepsilon=0$ in expression of controller (5), then we will have $\mathbf{z} \in \mathbb{R}^{n}$. As a result, the $(2,2)$ element in BMI (17a) (resp. BMI $(17 b))$ becomes $-2 \beta_{1} d+2 \beta_{2} l$ (resp. $\left.\left(p^{2} / \lambda\right)+2 \alpha_{1} d-2 \alpha_{2} l\right)$. As $d>0$ and $l<0$, then it follows that $-2 \beta_{1} d+2 \beta_{2} l<0$ and $\left(p^{2} / \lambda\right)+2 \alpha_{1} d-2 \alpha_{2} l>0$. Hence, the two BMIs (17a) and (17b) are unfeasible. Therefore, to solve such a feasibility problem, we add the constraint $\mathbf{z}^{T} \mathscr{C}^{T} \mathbf{z}>\varepsilon$ on the state-feedback controller and with $\varepsilon$ as a small enough positive parameter.

\section{Synthesis of the Robust State-Feedback Control Law: LMI Conditions}

It is worth mentioning that the four inequality conditions in (17a)-(17d) in Theorem 1 are BMIs. Thus, to solve the stability problem of the closed-loop impulsive system by finding the feedback matrix gain $\mathscr{K}$ of the state-feedback control law (5) and the corresponding Lyapunov matrix $\mathscr{P}$, we should transform these BMIs into LMIs.

Theorem 2. The impulsive hybrid systems (3a) and (3b) subject to the external disturbance $v(t)$, with $\|v(t)\| \leq p$, is robustly and asymptotically stabilizable by the state- feedback control law (5) if, for a scalar $0<\varepsilon \ll 1$ fixed a priori, there exist a symmetric matrix $\mathbf{S}$, a matrix $\mathscr{K}$, and positive scalars $\mu_{1}, \mu_{2}, \mu_{3}, \mu_{4}, \eta_{1}, \eta_{2}, \eta_{3}, \eta_{4}, \widehat{\xi}_{1}, \widehat{\xi}_{2}, \lambda$, and $\hat{p}$ such that the following LMI-based optimization problem is feasible:

maximize $\widehat{p}$

s.t.

$\left[\begin{array}{ccccc}\mathcal{S} & \mathcal{S} \mathscr{C} & \frac{1}{2 d} \mathscr{C} & -\frac{1}{2 l} \mathcal{S} \mathscr{C} & \mathcal{O} \\ (\star) & \epsilon \eta_{3} & \mathcal{O} & \mathcal{O} & \mathcal{O} \\ (\star) & (\star) & \frac{1}{2 d} \eta_{1} & \mathcal{O} & \eta_{3} \\ (\star) & (\star) & (\star) & -\frac{1}{2 l} \eta_{2} & \eta_{3} \\ (\star) & (\star) & (\star) & (\star) & \eta_{3}\end{array}\right]>0$, 
and the maximum bound of the disturbance is defined as follows:

$$
p=\frac{\widehat{p}}{\mu_{3}}
$$

Proof. We begin first by linearizing BMI (17a). Let us pose $\mathcal{S}=\mathscr{P}^{-1}$. We pre- and postmultiplying (17a) by the matrix $\operatorname{diag}(\mathcal{S}, 1)$. Thus, we obtain the following condition:

$$
\left[\begin{array}{cc}
\mathcal{S}-\beta_{3} \mathcal{S} \mathscr{C} \mathscr{C}^{T} \mathcal{S} & \left(\beta_{1}-\beta_{2}\right) \mathcal{S} \mathscr{C} \\
(*) & \varepsilon \beta_{3}-2 \beta_{1} d+2 \beta_{2} l
\end{array}\right]>0 .
$$

For simplicity, posing $\mathscr{N}_{1}=\left[\begin{array}{cc}\eta_{1} & 0 \\ (\star) & \eta_{2}\end{array}\right]$ with $\eta_{1}=\beta_{1}^{-1}$, $\eta_{2}=\beta_{2}^{-1}, \mathscr{Q}^{-1}=\left[\begin{array}{cc}2 d & 0 \\ 0 & -2 l\end{array}\right], \mathscr{G}=\left[\begin{array}{ll}\mathcal{S} \mathscr{C} & -\mathcal{S} \mathscr{C}\end{array}\right], \mathscr{L}=\left[\begin{array}{l}1 \\ 1\end{array}\right]$, and $\eta_{3}^{-1}=\varepsilon \beta_{3}$. Then, it is easy to show that $\varepsilon \beta_{3}-2 \beta_{1} d+$ $2 \beta_{2} l=\eta_{3}^{-1}-\mathscr{L}^{T}\left(\mathcal{Q} \mathscr{N}_{1}\right)^{-1} \mathscr{L}$ and $\left(\beta_{1}-\beta_{2}\right) \mathcal{S} \mathscr{C}=\mathscr{G} \mathscr{N}_{1}^{-1} \mathscr{L}$. Hence, the matrix inequality (27) becomes

$$
\left[\begin{array}{cc}
\mathcal{S}-\left(\varepsilon \eta_{3}\right)^{-1} \mathcal{S} \mathscr{C} \mathscr{C}^{T} \mathcal{S} & \mathscr{G} \mathscr{N}_{1}^{-1} \mathscr{L} \\
(\star) & \eta_{3}^{-1}-\mathscr{L}^{T}\left(\mathscr{Q} \mathcal{N}_{1}\right)^{-1} \mathscr{L}
\end{array}\right]>0 .
$$

Based on the Schur complement lemma (see Lemma 3), matrix (28) is equivalent to

$$
\begin{aligned}
\mathcal{S} & -\left(\varepsilon \eta_{3}\right)^{-1} \mathcal{S} \mathscr{C} \mathscr{C}^{T} \mathcal{S}-\mathscr{G} \mathscr{N}_{1}^{-1} \mathscr{L}\left(\eta_{3}^{-1}-\mathscr{L}^{T}\left(\boldsymbol{Q} \mathcal{N}_{1}\right)^{-1} \mathscr{L}\right)^{-1} \\
& \mathscr{L}^{T} \mathscr{N}_{1}^{-1} \mathscr{G}^{T}>0
\end{aligned}
$$$$
\eta_{3}^{-1}-\mathscr{L}^{T}\left(\mathscr{Q} \mathscr{N}_{1}\right)^{-1} \mathscr{L}>0
$$

The matrix inversion lemma (see Lemma 2) states that

$$
\left(\eta_{3}^{-1}-\mathscr{L}^{T}\left(\mathscr{Q} \mathscr{N}_{1}\right)^{-1} \mathscr{L}\right)^{-1}=\eta_{3}-\eta_{3}^{2} \mathscr{L}^{T} \mathscr{H}^{-1} \mathscr{L}
$$

with $\mathscr{H}=\eta_{3} \mathscr{L} \mathscr{L}^{T}-\mathscr{Q} \mathscr{N}_{1}$. If we consider in the next that $\mathscr{H}<0$, then since $\eta_{3}>0$, we obtain $\eta_{3}-\eta_{3}^{2} \mathscr{L}^{T} \mathscr{H}^{-1} \mathscr{L}>0$. Therefore, condition (29b) is always satisfied. We will only consider in the sequel condition (29a).

Substituting expression (30) in (29a) yields

$$
\begin{gathered}
\mathcal{S}-\left(\varepsilon \eta_{3}\right)^{-1} \mathcal{S} \mathscr{C} \mathscr{C}^{T} \mathcal{S}-\eta_{3} \mathscr{G} \mathscr{N}_{1}^{-1} \mathscr{L} \mathscr{L}^{T} \mathcal{N}_{1}^{-1} \mathscr{G}^{T} \\
+\eta_{3}^{2} \mathscr{G} \mathscr{N}_{1}^{-1} \mathscr{L} \mathscr{L}^{T} \mathscr{H}^{-1} \mathscr{L} \mathscr{L}^{T} \mathcal{N}_{1}^{-1} \mathscr{G}^{T}>0 .
\end{gathered}
$$

Relying on expression of the matrix $\mathscr{H}$, it is straightforward to demonstrate that

$$
\eta_{3} \mathscr{G} \mathscr{N}_{1}^{-1} \mathscr{L} \mathscr{L}^{T}=\mathscr{G} \mathscr{N}_{1}^{-1}\left(\mathscr{H}+\mathscr{Q} \mathscr{N}_{1}\right) .
$$

Thus, based on this relation (32) and as the matrices $\mathbb{Q}$ and $\mathscr{N}_{1}$ are diagonal and $\mathscr{H}$ is symmetric, we obtain the following expressions:

$$
\begin{aligned}
& \eta_{3} \mathscr{G} \mathcal{N}_{1}^{-1} \mathscr{L} \mathscr{L}^{T} \mathcal{N}_{1}^{-1} \mathscr{G}^{T}=\mathscr{G} \mathcal{N}_{1}^{-1} \mathscr{H} \mathcal{N}_{1}^{-1} \mathscr{G}^{T}+\mathscr{G} \mathscr{Q} \mathscr{N}_{1}^{-1} \mathscr{G}^{T}, \\
& \eta_{3}^{2} \mathscr{G} \mathcal{N}_{1}^{-1} \mathscr{L} \mathscr{L}^{T} \mathscr{H}^{-1} \mathscr{L} \mathscr{L}^{T} \mathcal{N}_{1}^{-1} \mathscr{G}^{T}=\mathscr{G} \mathscr{N}_{1}^{-1} \mathscr{H} \mathscr{N}_{1}^{-1} \mathscr{G}^{T} \\
& \quad+2 \mathscr{G} \mathcal{N}_{1}^{-1} \mathfrak{Q} \mathscr{G}^{T}+\mathscr{G} \mathbb{Q} \mathscr{H}^{-1} \mathfrak{Q} \mathscr{G}^{T} .
\end{aligned}
$$

By substituting the two expressions (33a) and (33b) in inequality (31), we obtain

$$
\mathcal{S}-\left(\varepsilon \eta_{3}\right)^{-1} \mathcal{S} \mathscr{C} \mathscr{C}^{T} \mathcal{S}+\mathscr{G} \mathscr{N}_{1}^{-1} \mathfrak{Q} \mathscr{G}^{T}+\mathscr{G} \mathscr{Q} \mathscr{H}^{-1} \mathfrak{Q} \mathscr{G}^{T}>0 .
$$

Moreover, as $\mathscr{N}_{1}^{-1} \mathfrak{Q}>0$, then condition (34) is satisfied if

$$
\mathcal{S}-\left(\varepsilon \eta_{3}\right)^{-1} \mathcal{S} \mathscr{C} \mathscr{C}^{T} \mathcal{S}+\mathscr{G} \mathscr{Q} \mathscr{H}^{-1} \mathfrak{Q} \mathscr{G}^{T}>0 .
$$

As $\varepsilon \eta_{3}>0$ and $\mathscr{H}<0$, then the Schur complement lemma states that the matrix inequality (35) is equivalent to

$$
\left[\begin{array}{ccc}
\mathcal{S} & \mathcal{S} \mathscr{C} & \mathscr{G} Q \\
(\star) & \varepsilon \eta_{3} & \mathscr{O} \\
(\star) & (\star) & \mathcal{N}_{1} \mathscr{Q}-\eta_{3} \mathscr{L}^{T}
\end{array}\right]>0
$$

By applying the Schur complement on elements (33a) and (33b) in (36), we obtain the following matrix inequality:

$$
\left[\begin{array}{cccc}
\mathcal{S} & \mathcal{S} \mathscr{C} & \mathscr{G} \mathscr{Q} & \mathcal{O} \\
(\star) & \varepsilon \eta_{3} & \mathcal{O} & \mathcal{O} \\
(\star) & (\star) & \mathcal{N}_{1} \mathscr{Q} & \eta_{3} \mathscr{L} \\
(\star) & (\star) & (\star) & \eta_{3}
\end{array}\right]>0 .
$$

Accordingly, by replacing the different matrices in (37) by their expression, we found the first LMI (24a).

We focus now on BMI (17b). Pre- and postmultiplying the matrix inequality $(17 \mathrm{~b})$ by the matrix $\operatorname{diag}(\mathcal{S}, 1)$ gives the following condition:

$$
\left[\begin{array}{cc}
(\mathscr{A} \mathcal{S}+\mathscr{B} \widehat{\mathscr{K}})+(*)+\lambda \mathscr{B} \mathscr{B}^{T}+\alpha_{3} \mathcal{S} \mathscr{C}_{\mathscr{C}^{T} \mathcal{S}} & -\left(\alpha_{1}-\alpha_{2}\right) \mathcal{S} \mathscr{C} \\
(*) & -\varepsilon \alpha_{3}+\frac{p^{2}}{\lambda}+2 \alpha_{1} d-2 \alpha_{2} l
\end{array}\right]<0 \text {, }
$$


where $\widehat{\mathscr{K}}=\mathscr{K} \mathcal{S}$.
$\quad$ For simplicity, posing $\mathscr{G}=\left[\begin{array}{lll}\mathcal{S} \mathscr{C} & -\mathcal{S} \mathscr{C} & \mathcal{O}\end{array}\right], \mathscr{L}=\left[\begin{array}{l}1 \\ 1 \\ 1\end{array}\right]$, $\mathscr{Q}^{-1}=\left[\begin{array}{ccc}2 d & 0 & 0 \\ 0 & -2 l & 0 \\ 0 & 0 & p^{2}\end{array}\right]$, and $\mathscr{N}_{2}=\left[\begin{array}{ccc}\mu_{1} & 0 & 0 \\ 0 & \mu_{2} & \\ 0 & 0 & \lambda\end{array}\right]$ with $\mu_{1}=\alpha_{1}^{-1}$, $\mu_{2}=\alpha_{2}^{-1}$, and $\mu_{3}^{-1}=\varepsilon \alpha_{3}$, it is easy to show that $-\varepsilon \alpha_{3}+\left(p^{2} / \lambda\right)+2 \alpha_{1} d-2 \alpha_{2} l=-\left(\mu_{3}^{-1}-\mathscr{L}^{T}\left(\mathscr{Q} \mathcal{N}_{2}\right)^{-1} \mathscr{L}\right)$

and $\left(\alpha_{1}-\alpha_{2}\right) \mathcal{S} \mathscr{C}=\mathscr{G} \mathscr{N}_{2}^{-1} \mathscr{L}$. It is worth to note that $\mathbb{Q}>0$ and $\mathcal{N}_{2}>0$.

Then, by multiplying the matrix inequality $(37)$ by $(-1)$, we obtain the following result:

$$
\left[\begin{array}{cc}
-\left((\mathscr{A} \mathcal{S}+\mathscr{B} \widehat{K})+(\star)+\lambda \mathscr{B} \mathscr{B}^{T}\right)-\left(\varepsilon \mu_{3}\right)^{-1} \mathcal{S} \mathscr{C} \mathscr{C}^{T} \mathcal{S} & \mathscr{G} \mathcal{N}_{2}^{-1} \mathscr{L} \\
(\star) & \mu_{3}^{-1}-\mathscr{L}^{T}\left(\mathscr{Q} \mathcal{N}_{2}\right)^{-1} \mathscr{L}
\end{array}\right]>0 .
$$

By following the same linearization methodology adopted previously, we obtain the following simplified equivalent conditions:

$$
\begin{aligned}
- & (\mathscr{A} \mathcal{S}+\mathscr{B} \widehat{\mathscr{K}})+(\star)-\lambda \mathscr{B} \mathscr{B}^{T}-\left(\varepsilon \mu_{3}\right)^{-1} \mathcal{S} \mathscr{C} \mathscr{C}^{T} \mathcal{S} \\
& +\mathscr{G} \mathscr{Q} \mathscr{H}^{-1} \mathscr{Q} \mathscr{G}^{T}>0, \\
\mathscr{H} & =\mu_{3} \mathscr{L} \mathscr{L}^{T}-\mathscr{Q} \mathscr{N}_{2}<0 .
\end{aligned}
$$

Since $\mathscr{H}<0$ and $\left(\varepsilon \mu_{3}\right)>0$, the Schur complement states that the matrix inequality (40a) is equivalent to

$$
\left[\begin{array}{cccc}
-(\mathscr{A} \mathcal{S}+\mathscr{B} \widehat{\mathscr{K}})+(\star)-\lambda \mathscr{B} \mathscr{B}^{T} & \mathcal{S} \mathscr{C} & \mathscr{G} \mathfrak{Q} & \mathcal{O} \\
(\star) & \varepsilon \mu_{3} & \mathcal{O} & \mathcal{O} \\
(\star) & (\star) & \mathcal{N}_{2} \mathfrak{Q} & \mu_{3} \mathscr{L} \\
(\star) & (\star) & (\star) & \mu_{3}
\end{array}\right]>0 .
$$

By multiplying the matrix inequality (41) from left and right by the matrix $\operatorname{diag}(\mathscr{I}, 1,1,1, p, 1)$ and by making a new variable change $\widehat{p}=\mu_{3} p$, we obtain then LMI (24b).

We focus now in the linearization of BMIs (17c) and (17d). It is clear that these two BMIs are similar. Thus, the linearization of BMI (17d) will be identical to that for BMI (17c). Then, we will only linearize the first BMI (17c).

The Schur complement states that the matrix inequality $(17 \mathrm{c})$ is equivalent to

$$
\begin{array}{r}
\mathscr{R} \mathscr{P} \mathscr{R}-\mathscr{P}-\xi_{1} \mathscr{C} \mathscr{C}^{T}+\alpha_{4} \mathscr{C}^{T} \\
+\xi_{1}^{2} d^{2}\left(\alpha_{4} \varepsilon+\xi_{1} d^{2}\right)^{-1} \mathscr{C} \mathscr{C}^{T}<0, \\
\alpha_{4} \varepsilon+\xi_{1} d^{2}>0 .
\end{array}
$$

The matrix inversion lemma states that

$$
\left(\alpha_{4} \varepsilon+\xi_{1} d^{2}\right)^{-1}=\left(\xi_{1} d^{2}\right)^{-1}-\left(\xi_{1} d^{2}\right)^{-2}\left(\left(\xi_{1} d^{2}\right)^{-1}+\left(\alpha_{4} \varepsilon\right)^{-1}\right)^{-1} .
$$

By considering this relation (43) in (42a), this inequality (42a) is simplified as follows:

$$
\mathscr{R} \mathscr{P} \mathscr{R}-\mathscr{P}+\alpha_{4} \mathscr{C} \mathscr{C}^{T}-\left(\xi_{1}^{-1}+\frac{d^{2}}{\alpha_{4} \varepsilon}\right)^{-1} \mathscr{C} \mathscr{C}^{T}<0 .
$$

We pre- and postmultiply inequality (44) from left and right by $\mathcal{S}$, we obtain

$$
\mathcal{S} \mathscr{R} \mathcal{S}^{-1} \mathscr{R} \mathcal{S}-\mathcal{S}+\alpha_{4} \mathcal{S} \mathscr{C} \mathscr{C}^{T} \mathcal{S}-\left(\xi_{1}^{-1}+\frac{d^{2}}{\alpha_{4} \mathcal{\varepsilon}}\right)^{-1} \boldsymbol{S} \mathscr{C} \mathscr{C}^{T} \mathcal{S}<0 .
$$

Let $\xi_{1}>0, \alpha_{4}>0$, and $\varepsilon>0$, then condition (42b) is always satisfied, and hence we have $\xi_{1}^{-1}+\left(d^{2} / \alpha_{4} \varepsilon\right)>0$. Then, it is straightforward to demonstrate by means of the Young relation that

$$
\begin{gathered}
-\left(\xi_{1}^{-1}+\frac{d^{2}}{\alpha_{4} \varepsilon}\right)^{-1} \mathcal{S} \mathscr{C} \mathscr{C}^{T} \mathcal{S} \leq-\mathcal{S} \mathscr{C} \mathscr{C}^{T}+(*) \\
+\left(\xi_{1}^{-1}+\frac{d^{2}}{\alpha_{4} \varepsilon}\right) \mathscr{C} \mathscr{C}^{T} .
\end{gathered}
$$

Therefore, condition (45) becomes

$$
\begin{aligned}
\mathcal{S} \mathscr{R} \mathcal{S}^{-1} \mathscr{R} \mathcal{S}-\mathcal{S}+\alpha_{4} \mathcal{S} \mathscr{C} \mathscr{C}^{T} \mathcal{S}-\mathcal{S} \mathscr{C} \mathscr{C}^{T}+(\star) \\
+\left(\xi_{1}^{-1}+\frac{d^{2}}{\alpha_{4} \mathcal{\varepsilon}}\right) \mathscr{C} \mathscr{C}^{T}<0 .
\end{aligned}
$$

The Schur complement lemma states inequality (47) is equivalent to

$$
\left[\begin{array}{ccc}
-\mathcal{S}-\mathcal{S} \mathscr{C} \mathscr{C}^{T}+(*)+\left(\xi_{1}^{-1}+\frac{d^{2}}{\alpha_{4} \varepsilon}\right) \mathscr{C}^{T} & \mathcal{S} \mathscr{C} & \mathcal{S} \mathscr{R} \\
(\star) & -\frac{1}{\alpha_{4}} & \mathcal{O} \\
(\star) & (*) & -\mathcal{S}
\end{array}\right]<0 .
$$

Posing $\mu_{4}=\alpha_{4}^{-1}$ and $\widehat{\xi}_{1}=\xi_{1}^{-1}$, then the matrix inequality (48) is converted into LMI (24c). Similarly, we will obtain LMI (24d) from BMI (17d). This ends the proof of Theorem 2. 
TABLE 1: Numerical results for different values of $\varepsilon$.

\begin{tabular}{lcr}
\hline$\varepsilon$ & $p$ & $\mathscr{K}$ \\
\hline 10 & 2.7829 & {$[-43.2211-13.4620]$} \\
5 & 2.7826 & {$[-41.6121-12.5971]$} \\
1 & 2.7834 & {$[-43.4863-13.6926]$} \\
0.5 & 2.7828 & {$[-41.5044-12.5713]$} \\
0.1 & 2.7832 & {$[-66.3664-12.3858]$} \\
0.091 & 2.7826 & {$[-112.0141-10.5564]$} \\
0.09 & $(!)$ & $(!)$ \\
\hline
\end{tabular}

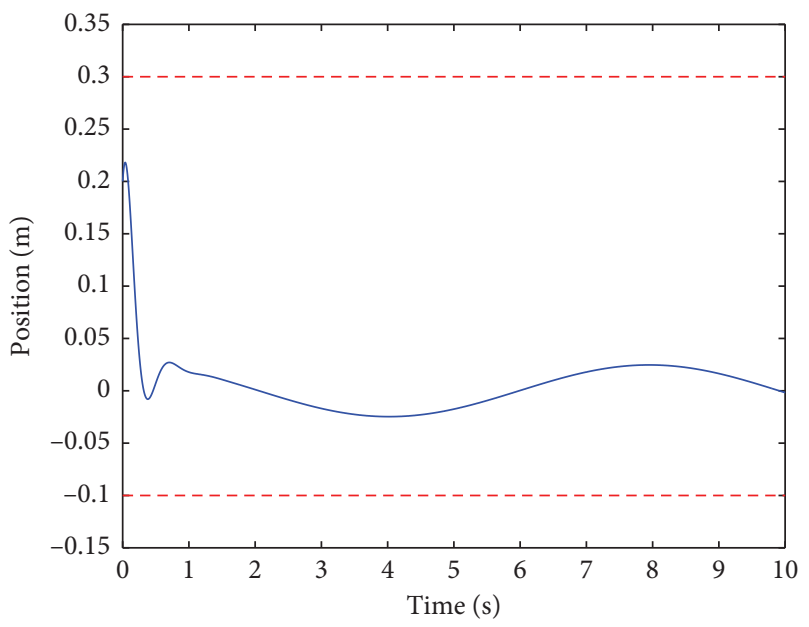

(a)

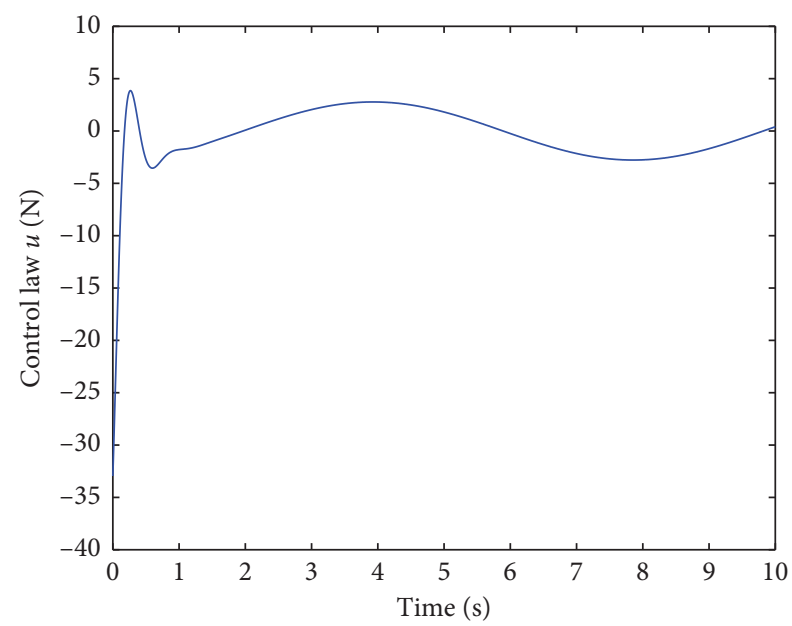

(c)

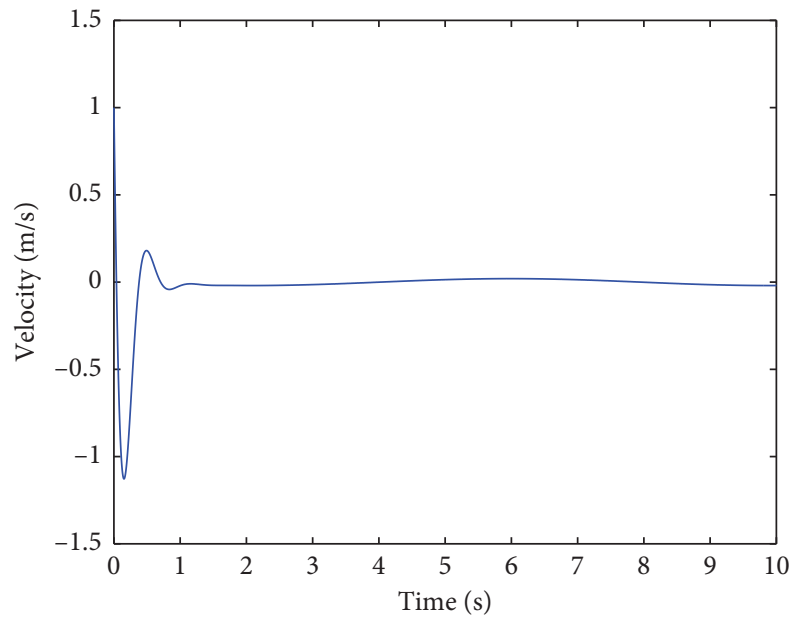

(b)

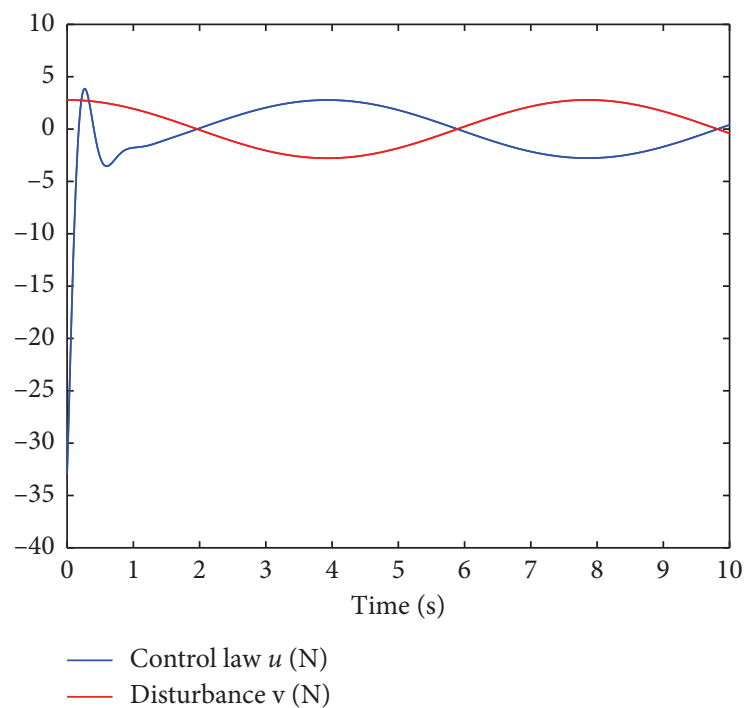

(d)

FIGURE 5: Simulation results of the two-sided asymmetric 1-DoF impact mechanical oscillator. Here, we fixed $d=0.3$.

\section{Simulation Results}

In the following simulation section, the parameters of the double-sided 1-DoF impact mechanical oscillator are fixed as follows: $m=1 \mathrm{~kg}, k=1 \mathrm{~N} / \mathrm{m}, c=0 \mathrm{~N} /(\mathrm{m} / \mathrm{s}), d=0.3 \mathrm{~m}$, $l=-0.1 \mathrm{~m}, r=0.8$, and $w=0.8 \mathrm{rad} \cdot \mathrm{s}^{-1}$. The numerical results of the optimization problem in Theorem 2 are given in Table 1 for different values of the parameter $\varepsilon$. In this table we provide the maximum bound $p$ of the disturbance $v$ and the corresponding controller gain $\mathscr{K}$. Notice that the symbol (!) in Table 1 means that optimization problem in Theorem 2 under LMIs (24a)-(24c) is unfeasible. Actually, it was found to be unfeasible for all $\varepsilon \leq 0.09$.

The first interesting and attractive observation that can be explored from Table 1 is that the size of the matrix gain $\mathscr{K}$ is very small. In addition, the second attractive result is that 


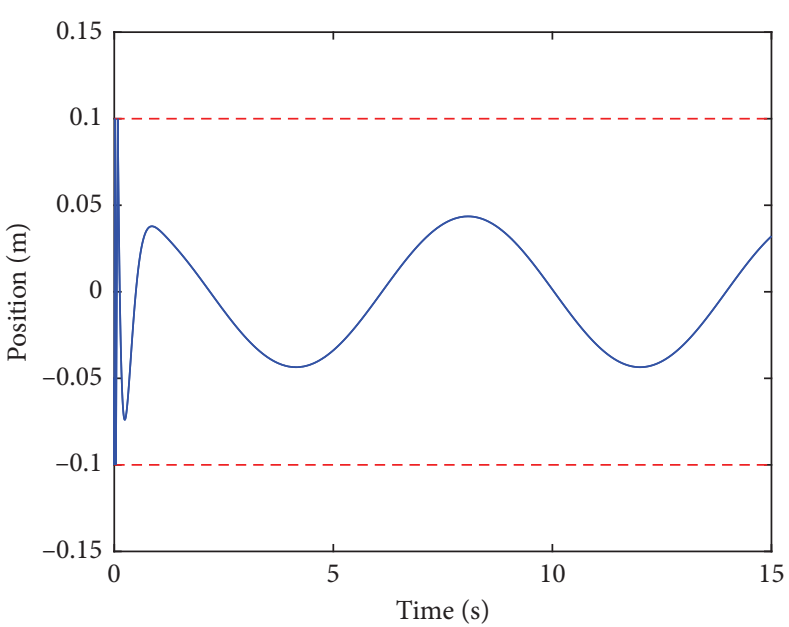

(a)

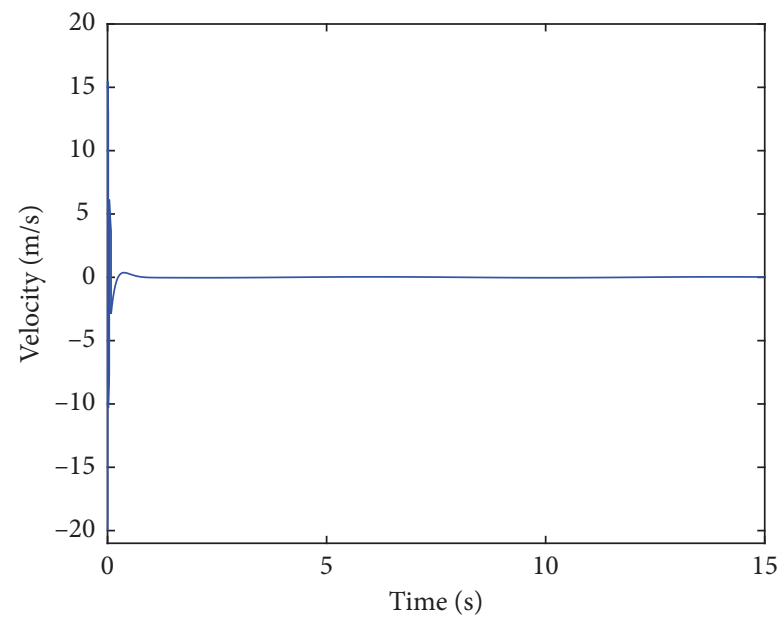

(c)

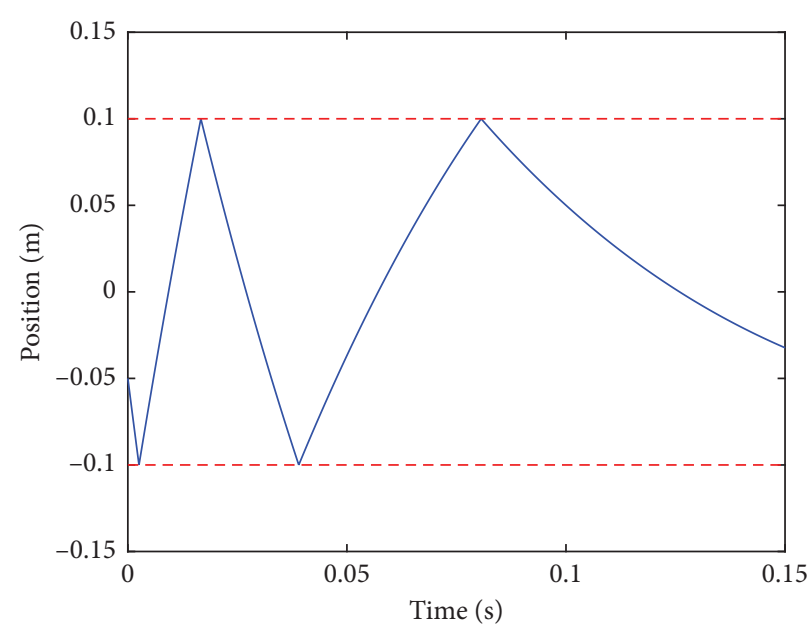

(b)

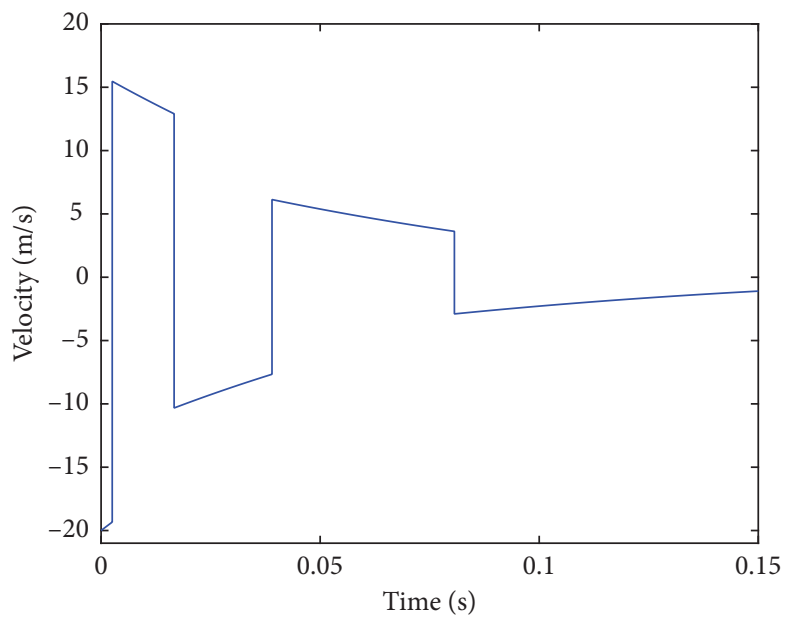

(d)

FIgURE 6: Simulation results of the two-sided asymmetric 1-DoF impact mechanical oscillator with $d=0.1$.

for all fixed values of the parameter $\varepsilon$ we obtained almost the same maximum bound $p$ of the disturbance signal $v(t)$, say about 2.78

Figure 5 show the simulation results for the impact oscillator using the values of $p$ and $\mathscr{K}$ obtained in Table 1 for $\varepsilon=0.091$. The initial condition adopted for the simulation is $\left[\begin{array}{ll}0.2 & 1\end{array}\right]^{T}$. Figure 5(a) reveals the temporal evolution of the position of the oscillator. Figure 5(b) shows it velocity. Figure 5(c) illustrates the applied controller $u$. In Figure 5(d), we transposed the control signal $u$ and the disturbance $v$. It is obvious that the mass's position of the impact oscillator will converge progressively in time to the zero-equilibrium point, around which the oscillator oscillates with a sinusoidal signal of a small amplitude 0.0247. In addition, the velocity oscillates around the zero state between \pm 0.0198 . According to this behavior, the controller will oscillate with a sinusoidal form of amplitude 2.774. Following this result and from Figure 5(d), we stress that the controller $u$ becomes opposite to the disturbance signal. Hence, this demonstrates that the controller $u$ has rejected approximately the persistent disturbance $v$.
Notice that for the adopted initial condition, the oscillator does not experience impacts with the left and the right end-stops. In order to observe the impacts with the two endstops, we modified the value of $d$ to be $d=0.1$ and we adopted another initial condition $\left[\begin{array}{ll}-0.05 & -20\end{array}\right]^{T}$. For this new value of the parameter $d$ and for $\varepsilon=0.091$, we obtained $p=$ 2.58 and $\mathscr{K}=[-57.9597-12.9790]$. The simulation results are given in Figures 6 and 7. In Figure 6(a), we plot the temporal evolution of the mass position of the impact oscillator. Figure 6(b) is an enlargement of Figure 6(a) for a simulation time between 0 and $0.15 \mathrm{~s}$. Figures $6(\mathrm{c})$ and $6(\mathrm{~d})$ show the variation of the velocity of the oscillator. Figure 7(a) reveals the temporal variation of the controller $u$. Figure 7(b) is a blow-up view of Figure 7(a). Figures 7(c) and 7(d) demonstrate the temporal evolution of the Lyapunov function $\mathscr{V}(\mathbf{z})$ defined by expression (15).

It is obvious from these simulation results that the mass of the oscillator experiences in the present case impacts with the two end-stops before its stabilization around the zeroequilibrium point. The amplitude of the position oscillation is almost 0.043 , whereas that of the velocity is about 0.035 . As 


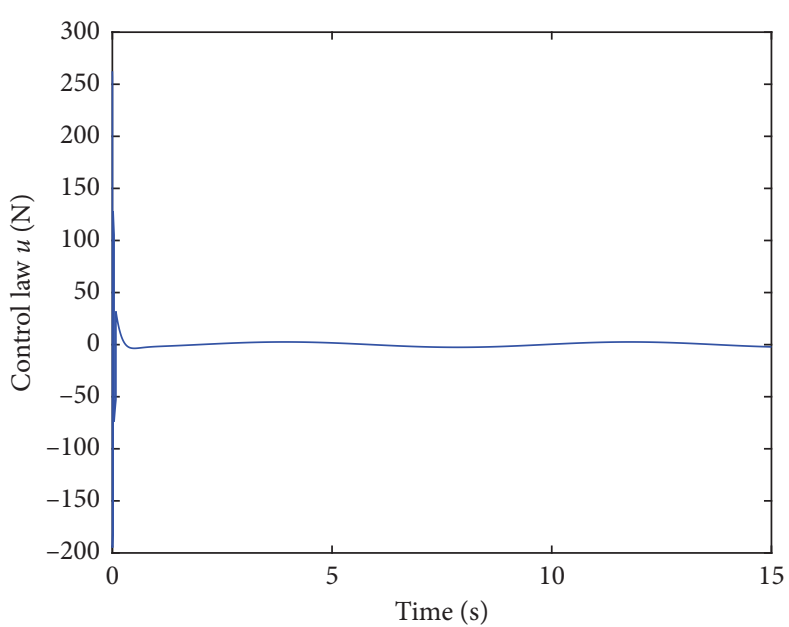

(a)

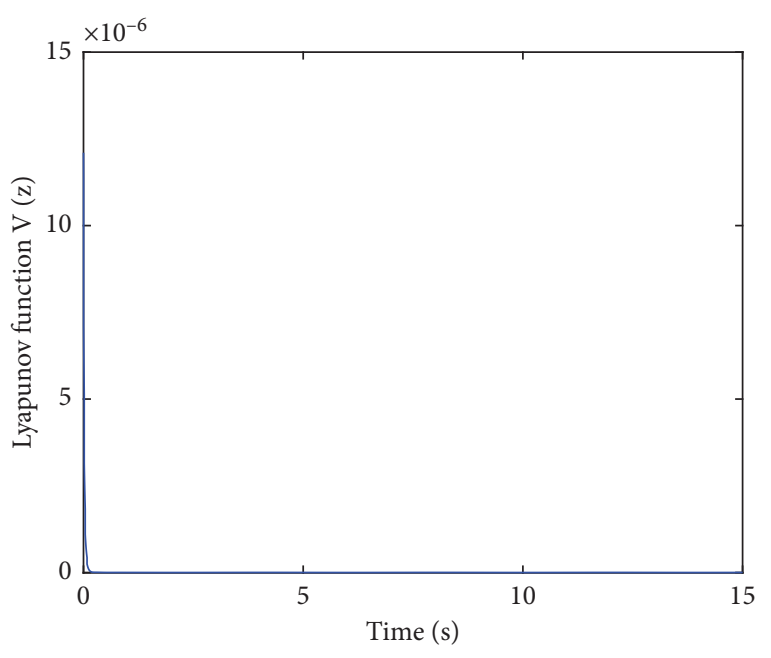

(c)

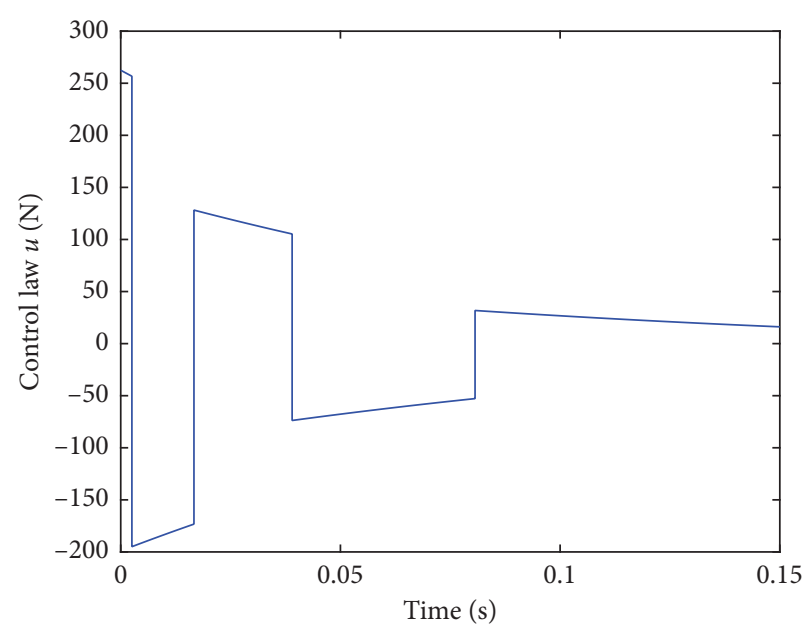

(b)

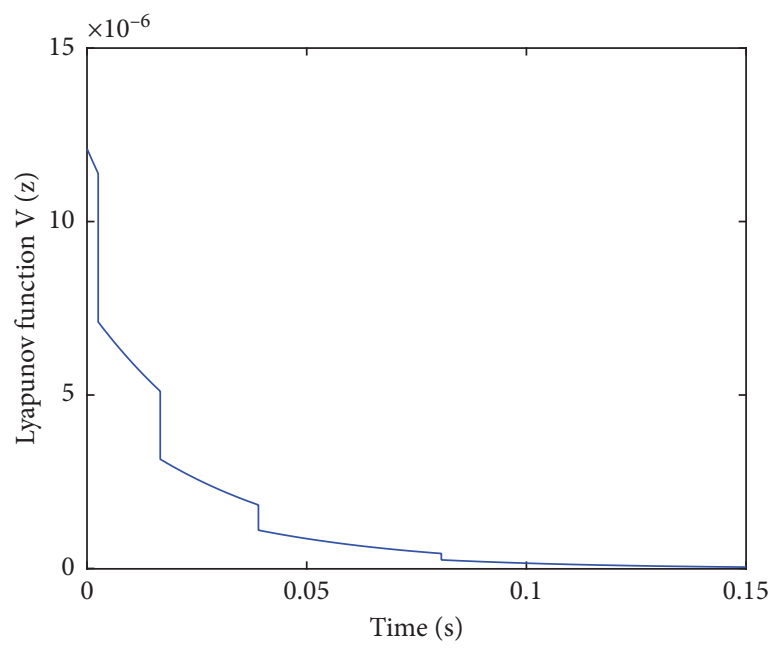

(d)

FIGURE 7: Simulation results for the case $d=0.1$ showing the temporal variation of the controller $u$ and the Lyapunov function $\mathscr{V}(\mathbf{z})$.

seen in Figure 6(d), at the impact with an end-stop, the velocity of the impact mechanical oscillator undergoes an instantaneous transition according to the restitution rule $\dot{\mathbf{x}}^{+}=-r \dot{\mathbf{x}}^{-}$. When an impact occurs, the Lyapunov function decreases instantaneously, as shown in Figure $7(\mathrm{c})$. This behavior reveals that the energy of the mechanical oscillator decreases at the impact events.

Remark 5. In the previous simulations, we considered a chaotic motion of the 1-DoF impact mechanical oscillator to be controlled. We can choose a regular motion of the uncontrolled mechanical system, and we will obtain the same results, that is, the robust control of the impact oscillator to the zero-equilibrium point despite the presence of the impacts with the two end-stops and also despite the presence of the external persistent disturbing torque. The choice of a chaotic behavior to be controlled can be explained by the fact that a chaotic motion is very sensitive to initial conditions and then its control is more "difficult" than a regular motion.

\section{Conclusion and Future Works}

In this paper, a robust control of the position of a two-sided 1-DoF impact mechanical oscillator under an unknown external disturbing torque by means of a state-feedback controller is proposed. Thus, the control problem of such an impact oscillator was formulated as a stabilization problem of an impulsive hybrid dynamics subject to state constraints. Our approach for the design of the stability conditions was based mainly on the use of the S-procedure lemma and also the Finsler lemma to transform the stability conditions under constraints into BMI conditions. We used the Schur complement lemma and the matrix inversion lemma to convert these BMIs into LMIs allowing the computation of the feedback gain and also the maximum bound of the disturbance. The simulation results show that the impact mechanical oscillator is robustly stabilized around the zeroequilibrium point.

Our future direction is to consider uncertainties in all the parameters of the impact oscillator. Thus, our objective is to design a robust controller for the impulsive hybrid dynamics 
under parametric uncertainties and disturbances. In addition, in this paper, we assumed that the position and the velocity of the oscillator are available for measurement. When this is not the case, we will consider the design of a static output feedback controller or an observer-based feedback controller to solve this problem. Another future work is to consider the problem of the impacting trajectory tracking, which is more difficult than that solved in the present paper.

\section{Data Availability}

The data used to support the findings of this study are available from the corresponding author upon request.

\section{Conflicts of Interest}

The authors declare that there are no conflicts of interest regarding the publication of this paper.

\section{References}

[1] R. A. Ibrahim, "Vibro-impact dynamics: modeling, mapping and applications," Lecture Notes in Applied and Computational Mechanics, Vol. 43, Springer-Verlag Berlin Heidelberg, Berlin, Germany, 1st Edition, 2009.

[2] Q. Tian, P. Flores, and H. M. Lankarani, "A comprehensive survey of the analytical, numerical and experimental methodologies for dynamics of multibody mechanical systems with clearance or imperfect joints," Mechanism and Machine Theory, vol. 122, pp. 1-57, 2018.

[3] P. Perlikowski, K.-C. Woo, S. Lenci, and T. Kapitaniak, "Special issue: dynamics of systems with impacts," Journal of Computational and Nonlinear Dynamics, vol. 16, no. 6, Article ID 060301, 2017.

[4] M. di Bernardo, C. Budd, A. R. Champneys, and P. Kowalczyk, "Piecewise-smooth dynamical systems: theory and applications," in Applied Mathematical Sciences, Vol. 163, Springer Science \& Business Media, New Work, NY, USA, 2008.

[5] D. J. Wagg and S. R. Bishop, "Chatter, sticking and chaotic impacting motion in a two-degree of freedom impact oscillator," International Journal of Bifurcation and Chaos, vol. 11, no. 1, pp. 57-71, 2001.

[6] L. S. Yousuf and D. B. Marghitu, "Experimental and simulation results of a cam and flat-faced follower mechanism," Journal of Computational and Nonlinear Dynamics, vol. 12, no. 6, Article ID 061001, 2017.

[7] M. Kapitaniak, V. Vaziri, J. Paez Chavez, and M. Wiercigroch, "Numerical study of forward and backward whirling of drillstring," Journal of Computational and Nonlinear Dynamics, vol. 12, no. 6, Article ID 061009, 2017.

[8] U. Andreaus, P. Baragatti, M. De Angelis, and S. Perno, "A preliminary experimental study about two-sided impacting SDOF oscillator under harmonic excitation," Journal of Computational and Nonlinear Dynamics, vol. 12, no. 6, Article ID 061010, 2017.

[9] U. Andreaus, P. Baragatti, M. De Angelis, and S. Perno, "Shaking table tests and numerical investigation of two-sided damping constraint for end-stop impact protection," Nonlinear Dynamics, vol. 90, no. 4, pp. 2387-2421, 2017.

[10] L. Guofang, W. Ding, and S. Wu, "Global behavior of vibroimpact system with multiple non-smooth mechanical factors," Journal of Computational and Nonlinear Dynamics, vol. 12, no. 6, Article ID 061004, 2017.

[11] Y. Yan, Y. Liu, and M. Liao, "A comparative study of the vibro-impact capsule systems with one-sided and two-sided constraints," Nonlinear Dynamics, vol. 89, no. 2, pp. 10631087, 2017.

[12] U. Andreaus and M. De Angelis, "Nonlinear dynamic response of a base-excited SDOF oscillator with double-side unilateral constraints," Nonlinear Dynamics, vol. 84, no. 3, pp. 1447-1467, 2016.

[13] H. Gritli and S. Belghith, "Diversity in the nonlinear dynamic behavior of a one-degree-of-freedom impact mechanical oscillator under OGY-based state-feedback control law: order, chaos and exhibition of the border-collision bifurcation," Mechanism and Machine Theory, vol. 124, pp. 1-41, 2018.

[14] U. Andreaus and M. De Angelis, "Experimental and numerical dynamic response of a SDOF vibro-impact system with double gaps and bumpers under harmonic excitation," International Journal of Dynamics and Control, vol. 7, no. 4, pp. 1278-1292, 2019.

[15] U. Andreaus and P. Casini, "Friction oscillator excited by moving base and colliding with a rigid or deformable obstacle," International Journal of Non-linear Mechanics, vol. 37, no. 1, pp. 117-133, 2002.

[16] K. Witkowski, G. Kudra, G. Wasilewski, and J. Awrejcewicz, "Modelling and experimental validation of 1-degree-offreedom impacting oscillator," Proceedings of the Institution of Mechanical Engineers, Part I: Journal of Systems and Control Engineering, vol. 233, no. 4, pp. 418-430, 2019.

[17] S. Skurativskyi, G. Kudra, G. Wasilewski, and J. Awrejcewicz, "Properties of impact events in the model of forced impacting oscillator: experimental and numerical investigations," International Journal of Non-linear Mechanics, vol. 113, pp. 55-61, 2019.

[18] F. Casas and C. Grebogi, "Control of chaotic impacts," International Journal of Bifurcation and Chaos, vol. 7, no. 4, pp. 951-955, 1997.

[19] S. R. Bishop, D. J. Wagg, and D. Xu, "Use of control to maintain period-1 motions during wind-up or wind-down operations of an impacting driven beam," Chaos, Solitons \& Fractals, vol. 9, no. 1-2, pp. 261-269, 1998.

[20] S. L. T. de Souza and I. L. Caldas, "Controlling chaotic orbits in mechanical systems with impacts," Chaos, Solitons \& Fractals, vol. 19, no. 1, pp. 171-178, 2004.

[21] H. Gritli, S. Belghith, and N. Khraief, "Chaos control of an impact mechanical oscillator based on the OGY method," in Handbook of Research on Advanced Intelligent Control Engineering and Automation, Ch. 9, A. T. Azar and S. Vaidyanathan, Eds., pp. 259-278, IGI Global, Hershey, PA, USA, 2015.

[22] C. J. Begley and L. N. Virgin, "On the ogy control of an impact-friction oscillator," Journal of Vibration and Control, vol. 7, no. 6, pp. 923-931, 2001.

[23] J. Awrejcewicz, K. Tomczak, and C.-H. Lamarque, "Controlling systems with impacts," International Journal of Bifurcation and Chaos, vol. 9, no. 3, pp. 547-553, 1999.

[24] G. W. Luo and X. H. Lv, "Controlling bifurcation and chaos of a plastic impact oscillator," Nonlinear Analysis: Real World Applications, vol. 10, no. 4, pp. 2047-2061, 2009.

[25] S. L. T. de Souza, I. L. Caldas, and R. L. Viana, "Damping control law for a chaotic impact oscillator," Chaos, Solitons \& Fractals, vol. 32, no. 2, pp. 745-750, 2007.

[26] E. Gutiérrez and D. K. Arrowsmith, "Control of a double impacting mechanical oscillator using displacement 
feedback," International Journal of Bifurcation and Chaos, vol. 14, no. 9, pp. 3095-3113, 2004.

[27] H. Xu, S. Yin, G. Wen, S. Zhang, and Z. Lv, "Discrete-in-time feedback control of near-grazing dynamics in the two-degreeof-freedom vibro-impact system with a clearance," Nonlinear Dynamics, vol. 87, no. 2, pp. 1127-1137, 2017.

[28] J.-Y. Lee and J.-J. Yan, "Position control of double-side impact oscillator," Mechanical Systems and Signal Processing, vol. 21, no. 2, pp. 1076-1083, 2007.

[29] H. Gritli and S. Belghith, "Master-slave controlled synchronization to control chaos in an impact mechanical oscillator," in Proceedings of the 12th International Multi-Conference on Systems, Signals Devices (SSD), pp. 1-6, Mahdia, Tunisia, March 2015.

[30] F. Turki, H. Gritli, and S. Belghith, "A linear matrix inequality approach for the position control of a double-side impact mechanical oscillator via a state feedback law," in Proceedings of the 2018 International Conference on Advanced Systems and Electric Technologies (IC_ASET), pp. 118-124, Hammamet, Tunisia, March 2018.

[31] H. Gritli, F. Turki, and S. Belghith, "State-feedback control via LMI approach of a 1-DoF disturbed impacting mechanical oscillator under double-side rigid constraints," in Proceedings of the 2018 International Conference on Advanced Systems and Electric Technologies (IC_ASET), pp. 441-448, Hammamet, Tunisia, March 2018.

[32] F. Turki, H. Gritli, and S. Belghith, "Robust feedback control of a mechanical system under double-side constraints using LMIs and ellipsoidal sets," in Proceedings of the 2018 IEEE 15th International Multi-Conference on Systems, Signals Devices (SSD15), pp. 1308-1314, Hammamet, Tunisia, March 2018.

[33] H. Gritli and S. Belghith, "Robust feedback control of the underactuated Inertia Wheel Inverted Pendulum under parametric uncertainties and subject to external disturbances: LMI formulation," Journal of The Franklin Institute, vol. 355, no. 18, pp. 9150-9191, 2018.

[34] H. Gritli, N. Khraief, A. Chemori, and S. Belghith, "Selfgenerated limit cycle tracking of the underactuated inertia wheel inverted pendulum under IDA-PBC," Nonlinear Dynamics, vol. 89, no. 3, pp. 2195-2226, 2017.

[35] S. L. T. de Souza and I. L. Caldas, "Calculation of Lyapunov exponents in systems with impacts," Chaos, Solitons \& Fractals, vol. 19, no. 3, pp. 569-579, 2004.

[36] K. Czolczynski, A. Okolewski, and B. Blazejczyk-Okolewska, "Lyapunov exponents in discrete modelling of a cantilever beam impacting on a moving base," International Journal of Non-linear Mechanics, vol. 88, no. Supplement C, pp. 74-84, 2017.

[37] W. Serweta, A. Okolewski, B. Blazejczyk-Okolewska, K. Czolczynski, and T. Kapitaniak, "Lyapunov exponents of impact oscillators with Hertz's and Newton's contact models," International Journal of Mechanical Sciences, vol. 89, pp. 194-206, 2014.

[38] W. Serweta, A. Okolewski, B. Blazejczyk-Okolewska, K. Czolczynski, and T. Kapitaniak, "Mirror hysteresis and Lyapunov exponents of impact oscillator with symmetrical soft stops," International Journal of Mechanical Sciences, vol. 101-102, pp. 89-98, 2015.

[39] P. C. Müller, "Calculation of lyapunov exponents for dynamic systems with discontinuities," Chaos, Solitons \& Fractals, vol. 5, no. 9, pp. 1671-1681, 1995.

[40] H. Gritli and S. Belghith, "Computation of the Lyapunov exponents in the compass-gait model under OGY control via a hybrid Poincaré map," Chaos, Solitons \& Fractals, vol. 81, pp. 172-183, 2015.

[41] H. Gritli and S. Belghith, "Bifurcations and chaos in the semipassive bipedal dynamic walking model under a modified OGY-based control approach," Nonlinear Dynamics, vol. 83, no. 4, pp. 1955-1973, 2016.

[42] S. Boyd, L. El-Ghaoui, E. Feron, and V. Balakrishnan, "Linear matrix inequalities in system and control theory," in Studies in Applied and Numerical Mathematics, vol. 15, SIAM, Philadelphia, PA, USA, 1st edition, 1994.

[43] P. Kohan-Sedgh, A. Khayatian, and M. H. Asemani, "Conservatism reduction in simultaneous output feedback stabilisation of linear systems," IET Control Theory \& Applications, vol. 10, no. 17, pp. 2243-2250, 2016. 


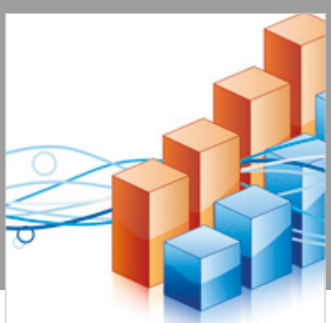

Advances in

Operations Research

\section{-n-m}
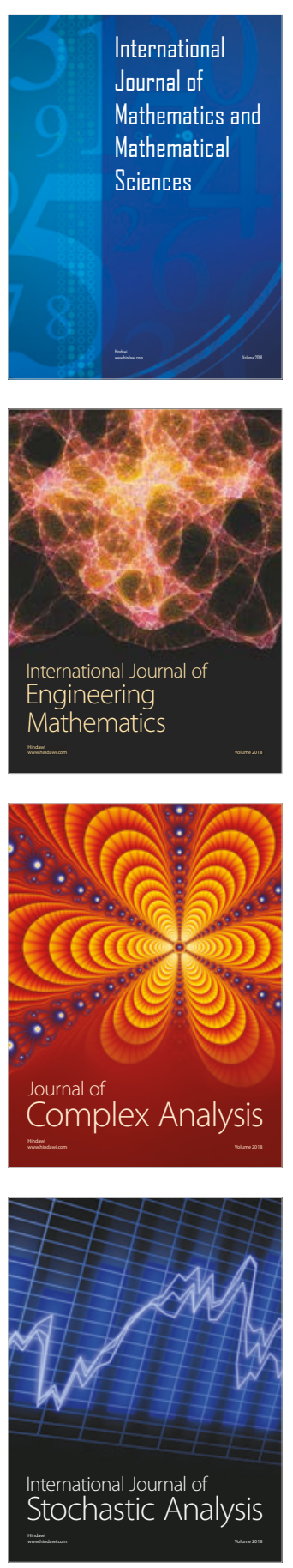
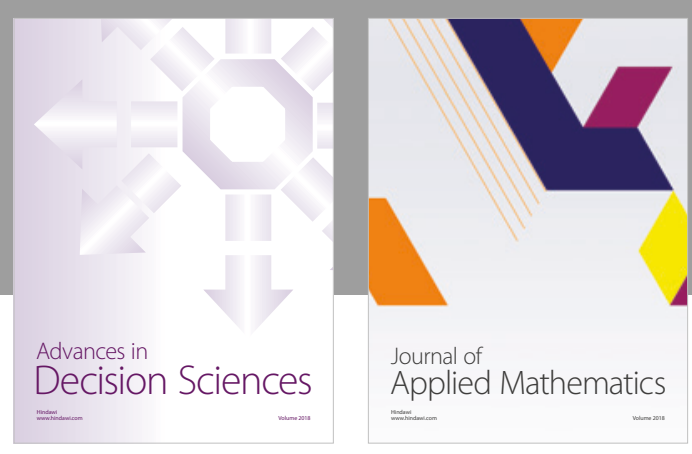

Journal of

Applied Mathematics
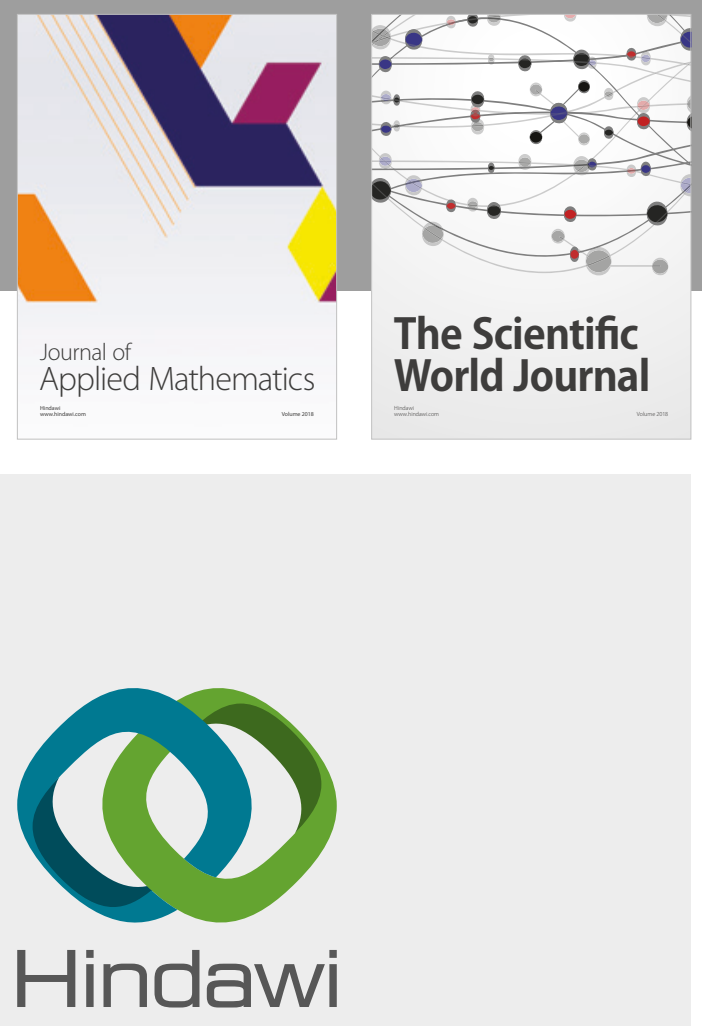

Submit your manuscripts at

www.hindawi.com

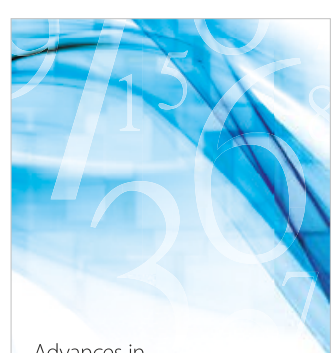

Advances in
Numerical Analysis
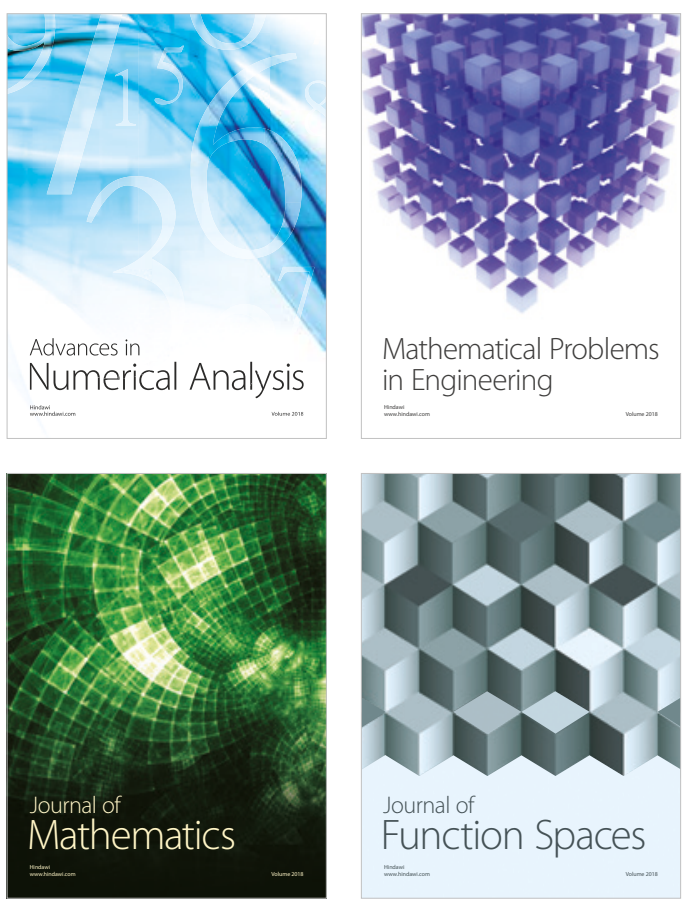

Mathematical Problems in Engineering

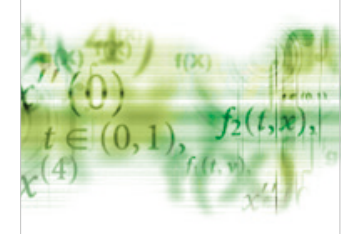

International Journal of

Differential Equations

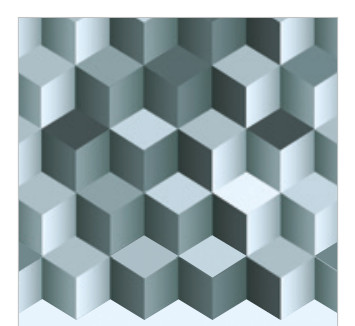

Journal of

Function Spaces
The Scientific

World Journal

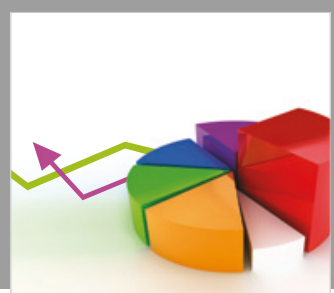

Journal of

Probability and Statistics
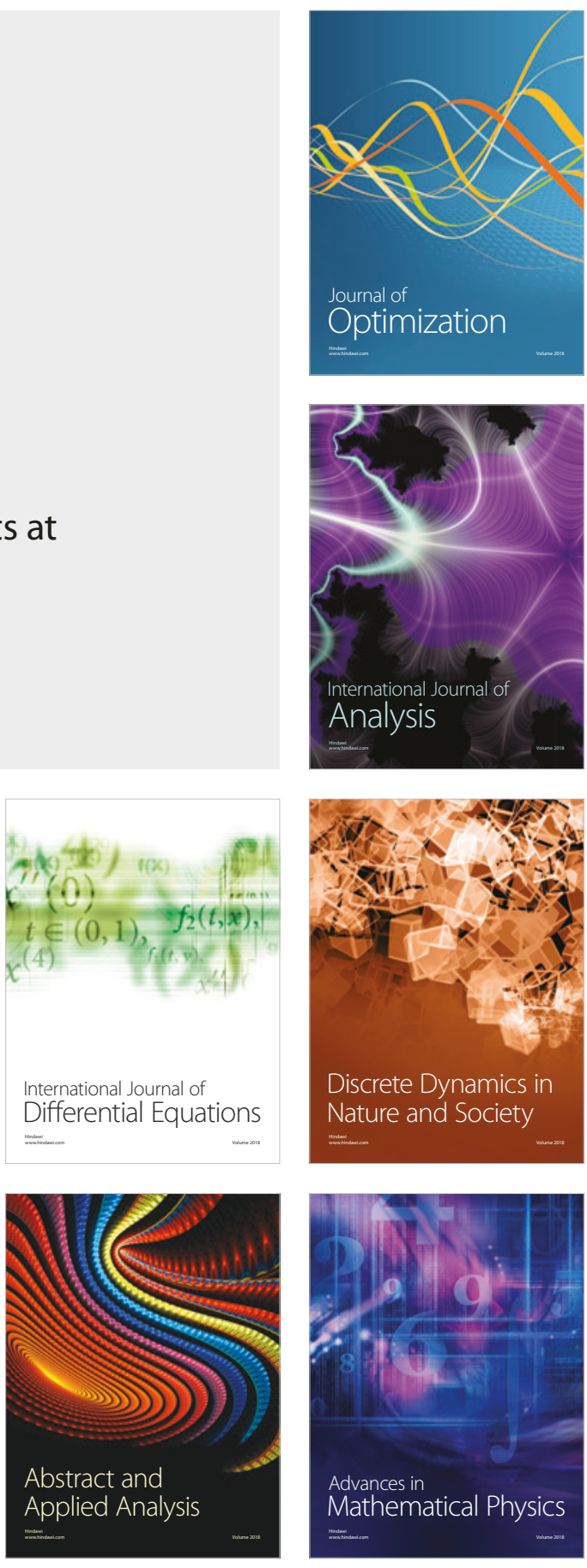